\title{
Circ-GALNT16 restrains colorectal cancer progression by enhancing the SUMOylation of hnRNPK
}

Chaofan Peng ${ }^{1,2,3 \dagger}$, Yuqian Tan ${ }^{1,2,3 \dagger}$, Peng Yang ${ }^{1,2,3 \dagger}$, Kangpeng Jin ${ }^{1,2,3 \dagger}$, Chuan Zhang ${ }^{1,2,3 \dagger}$, Wen Peng ${ }^{1,2,3}$, Lu Wang ${ }^{1,2,3}$, Jiahui Zhou ${ }^{1,2,3}$, Ranran Chen ${ }^{1,2,3}$, Tuo Wang ${ }^{1,2,3}$, Chi Jin ${ }^{1,2,3}$, Jiangzhou Ji, ${ }^{1,2,3}$, Yifei Feng ${ }^{1,2,3}$, Junwei Tang ${ }^{1,2,3^{*}}$ and Yueming Sun ${ }^{1,2,3^{*}}$

\begin{abstract}
Background: Recent studies have investigated the role of circular RNAs (circRNAs) as significant regulatory factors in multiple cancer progression. Nevertheless, the biological functions of circRNAs and the underlying mechanisms by which they regulate colorectal cancer (CRC) progression remain unclear.

Methods: A novel circRNA (circ-GALNT16) was identified by microarray and GRT-PCR. A series of in vitro and in vivo phenotype experiments were performed to investigate the role of circ-GALNT16 in CRC. The FISH, RNA pulldown assay, RIP assay, RNA sequencing, coimmunoprecipitation, and ChIP were performed to investigate the molecular mechanisms of circ-GALNT16 in CRC progression.

Results: Circ-GALNT16 was downregulated in CRC and was negatively correlated with poor prognosis. Circ-GALN T16 suppressed the proliferation and metastatic ability of CRC cells in vitro and in vivo. Mechanistically, circ-GALNT16 could bind to the $\mathrm{KH} 3$ domain of heterogeneous nuclear ribonucleoprotein $\mathrm{K}$ (hnRNPK), which promoted the SUMOylation of hnRNPK. Additionally, circ-GALNT16 could enhance the formation of the hnRNPK-p53 complex by facilitating the SUMOylation of hnRNPK. RNA sequencing assay identified serpin family E member 1 as the target gene of circ-GALNT16 at the transcriptional level. Rescue assays revealed that circ-GALNT16 regulated the expression of Serpine1 by inhibiting the deSUMOylation of hnRNPK mediated by SUMO-specific peptidase 2 and then regulating the sequence-specific DNA binding ability of the hnRNPK-p53 transcriptional complex.

Conclusions: Circ-GALNT16 suppressed CRC progression by inhibiting Serpine1 expression through regulating the sequence-specific DNA binding ability of the SENP2-mediated hnRNPK-p53 transcriptional complex and might function as a biomarker and therapeutic target for CRC.
\end{abstract}

Keywords: Colorectal cancer, circ-GALNT16, hnRNPK, SUMOylation, p53, Serpine1, SENP2

\footnotetext{
*Correspondence: pepsitjw@njmu.edu.cn; sunyueming@njmu.edu.cn

${ }^{+}$Chaofan Peng, Yuqian Tan, Peng Yang, Kangpeng Jin and Chuan Zhang contributed equally to this study.

'Department of General Surgery, The First Affiliated Hospital of Nanjing Medical University, Jiangsu 210029 Nanjing, People's Republic of China

Full list of author information is available at the end of the article
}

C C The Author(s). 2021 Open Access This article is licensed under a Creative Commons Attribution 4.0 International License, which permits use, sharing, adaptation, distribution and reproduction in any medium or format, as long as you give appropriate credit to the original author(s) and the source, provide a link to the Creative Commons licence, and indicate if changes were made. The images or other third party material in this article are included in the article's Creative Commons licence, unless indicated otherwise in a credit line to the material. If material is not included in the article's Creative Commons licence and your intended use is not permitted by statutory regulation or exceeds the permitted use, you will need to obtain permission directly from the copyright holder. To view a copy of this licence, visit http://creativecommons.org/licenses/by/4.0/ The Creative Commons Public Domain Dedication waiver (http://creativecommons.org/publicdomain/zero/1.0/) applies to the data made available in this article, unless otherwise stated in a credit line to the data. 


\section{Background}

Colorectal cancer (CRC) is one of the most common malignant tumors and continues to remain a public health concern [1]. The incidence and mortality rate of CRC rank third and second, respectively, among various cancers according to the up-to-date global cancer statistics. The prognoses of patients with advanced CRC remain poor, although neoadjuvant chemoradiotherapy, surgery, postoperative chemoradiotherapy, and immunotherapy are widely used to treat patients with CRC and have been gaining recognition rapidly [2]. One of the main causes for poor prognosis is that the detailed mechanisms of CRC progression remain unclear. Therefore, it is crucial to determine the unknown pathogenic mechanisms of CRC progression to develop specific diagnostic markers and accurate therapeutic targets.

Circular RNA (circRNA) is a kind of transcript that originates from the back-splicing of pre-mRNA [3]. CircRNAs have higher stability than linear transcripts and are more resistant to RNase $\mathrm{R}$ because of their loop structure, which makes circRNAs potential diagnostic markers and therapeutic targets for cancers $[4,5]$. The biological functions of circRNAs are widely involved in the proliferation, metastasis, apoptosis, and autophagy of various cancers through multiple pathways [6-9]. For example, hsa_circ_101555 can promote CRC progression by acting as a competing endogenous RNA of miR-597$5 p$ [10]. Circ-ABCC1 binds to $\beta$-catenin and activates the Wnt/ $\beta$-catenin pathway to facilitate $C R C$ progression [11]. CircPPP1R12A encodes a novel protein that promotes colon cancer pathogenesis through Hippo-YAP signaling [12]. However, the detailed mechanisms by which circRNAs regulate the progression of CRC remain elusive.

Heterogeneous nuclear ribonucleoprotein K (hnRNPK) is a DNA- and RNA-binding protein that regulates several biological processes, including transcriptional regulation, nucleocytoplasmic transport, and DNA damage repair [13]. Some studies on cancer have identified hnRNPK as an oncogene because of its overexpression in several cancer tissues, while hnRNPK has also been identified as an antioncogene as it activates the p53 pathway. SUMOylation is a type of posttranslational modification of hnRNPK by conjugating a small ubiquitin-like modifier (SUMO) covalently through a multistep reaction [14]. The SUMOylation of hnRNPK may regulate RNA metabolism at the transcriptional and posttranscriptional levels [15]. Several studies have reported that the SUMOylation of hnRNPK could enhance the interaction between hnRNPK and p53 and regulate the transcriptional activity of p53 [16-18].

In the present study, we elucidated whether circGALNT16 could suppress CRC progression by enhancing the SUMOylation of hnRNPK to regulate the sequence-specific DNA binding ability of the hnRNPKp53 complex. Collectively, our study investigated circGALNT16-mediated specific molecular mechanisms in CRC progression, which might contribute to determining the suitability of circ-GALNT16 as a potential diagnostic marker and therapeutic target for CRC.

\section{Methods}

\section{Microarray analysis of circRNAs}

The circRNA microarray analysis (CapitalBio Technology, Beijing, China) was performed using specific probes targeting the back-splicing sites of human circRNAs. The circRNAs were detected in five pairs of CRC tissues. Data normalization, summarization, and quality control were performed by the GeneSpring software. The differential circRNAs are listed in Table S1. All the raw data of the microarray analysis are available in the Gene Expression Omnibus (accession number: GSE172229).

\section{Patient samples and cell culture}

A total of 100 tumor samples from patients with CRC and paired adjacent normal tissues were collected between 2014 and 2018 from the Department of General Surgery, First Affiliated Hospital of Nanjing Medical University, Nanjing, China. Fresh tissues were obtained after surgery and immediately frozen at $-80{ }^{\circ} \mathrm{C}$. Two $\mathrm{ml}$ RNAlater (AM7020, Thermo Fisher Scientific, Waltham, USA) was used to protect the tissue RNA from degradation. Patients who had received neoadjuvant chemoradiotherapy were excluded. This study was approved by the Human Ethics Committee of First Affiliated Hospital of Nanjing Medical University. Each patient was informed about the study and consented to specimen donation before the surgery. The raw data of circ-GALN T16 expression in the 100 pairs of CRC tissues and relative expression in different pathological subgroups are provided in Table S2.

The cell lines LoVo, DLD-1, SW-480, Caco-2, RKO, HCT 116, HT-29, and NCM460 were purchased from the Cell Bank of Type Culture Collection of the Chinese Academy of Sciences (Shanghai, China) and cultured in a recommended medium with $10 \%$ fetal bovine serum at $37{ }^{\circ} \mathrm{C}$ in a $5 \% \mathrm{CO}_{2}$ humidified incubator.

\section{RNA extraction and qRT-PCR}

Total RNA was extracted from patients' specimens and cells by TRIzol reagent (Invitrogen, USA), and the extraction procedure was performed as described previously [19]. Then, a PrimeScript RT reagent kit (TaKara, Dalian, China) was used for reverse transcription. An SYBR Premix Ex Taq Kit (TaKara) was used for qRTPCR on an Applied Biosystems 7500 Sequence Detection System. GAPDH was used as an internal control. The sequences of primers are listed in Table S3. 


\section{Cell transfection}

A lentivirus containing short hairpin RNAs (shRNAs) and full-length targeting sequence of circ-GALNT16, including the corresponding negative control, were synthesized by Obio (Shanghai, China). The Serpine1 overexpression plasmid was obtained from Obio. The truncated hnRNPK plasmids of hnRNPK with a Cterminus $3 \times$ Flag tag were synthesized by Obio. The small interfering RNA (siRNA) oligonucleotides targeting hnRNPK, SENP2, and Serpine1 were synthesized by RiboBio (Guangzhou, China). The transfection procedures were performed as described previously by using Lipofectamine 3000 (Invitrogen) [20]. The sequences of shRNAs and siRNAs are listed in Table S3.

\section{Cell proliferation assays}

Cell proliferation assays, including Cell Counting Kit-8 assay and colony formation assay, were performed as described previously [21]. The cell proliferation ability was measured by the 5-ethynyl-2 -deoxyuridine assay using an EdU kit (Beyotime, Shanghai, China).

\section{Transwell and scratch wound healing assay}

Transwell assay was performed with Millipore cell culture inserts (24-well insert, $8 \mu \mathrm{m}$ pore size). For migration assay, $3 \times 10^{4}$ cells resuspended in $200 \mu \mathrm{L}$ serumfree medium per well were seeded onto the upper chamber of the Transwell membrane, while the lower chamber was filled with $700 \mu \mathrm{L}$ culture medium containing $10 \%$ serum. After $24 \mathrm{~h}$ (36 or $48 \mathrm{~h}$ based on different cell lines), we stained the cells adhering to the underside of the membrane by using a Crystal Violet solution and removed the cells above the membrane with swabs. Cells in each well were counted by five random views under a microscope. For invasion assay, Matrigel (BD Biosciences, Franklin Lakes, NJ, USA) was spread on the upper layer, and the remaining steps were performed as described above.

A $200-\mu \mathrm{L}$ pipette tip was used to produce consistent length lesions in 6 -well plates $\left(8 \times 10^{5}\right.$ cells per well) for wound healing assay. An inversion microscope was used to acquire each wound image at 0 and $48 \mathrm{~h}$. We used ImageJ software to quantitatively evaluate the gap distance.

\section{Flow cytometry assay of cell cycle and apoptosis}

Treated cells were fixed in $75 \%$ alcohol overnight at $-20{ }^{\circ} \mathrm{C}$ for cell cycle assay. The cells were washed three times, and a Cell Cycle Analysis Kit (Beyotime, Shanghai, China) was used for propidium iodide (PI) staining.

For apoptosis analysis, all cells were treated with 0.5 $\mathrm{mM} \mathrm{H}_{2} \mathrm{O}_{2}$ for $4 \mathrm{~h}$ to stimulate apoptosis. An Annexin VAPC/7-AAD Apoptosis Detection Kit (KeyGEN, Jiangsu, China) was used for Annexin V-APC and 7-
AAD staining according to the manufacturer's protocol. Finally, the cell cycle distribution percentage and the apoptotic rate were analyzed using BD FACSCanto II (BD Biosciences, San Jose, CA, USA).

\section{Animal models}

Four-week-old male BALB/c nude mice were purchased from the Animal Center of Nanjing Medical University (Nanjing, China) for subcutaneous tumor formation and liver metastasis model. For the xenograft model, $10^{6}$ DLD-1 cells transfected with sh-circ-GALNT16\#1 and RKO cells transfected with circ-GALNT16 as well as corresponding control cells were resuspended in $1 \mathrm{~mL}$ phosphate-buffered saline (PBS) and subcutaneously injected into the left and right armpits of the mice, respectively. The volume of each tumor was calculated every 5 days since the tumor became macroscopic. All the mice were sacrificed 30 days after the subcutaneous injection, and the xenograft tumors were weighed and subjected to immunohistochemistry (IHC).

A total of $10^{6}$ cells suspended in $20 \mu \mathrm{L}$ PBS were injected into the distal tip of the spleen of mice to establish the metastatic model. The liver tissues were dissected and embedded in paraffin for hematoxylin and eosin (H\&E) staining 6 weeks later. All the animal experiments were ratified by the Committee on the Ethics of Animal Experiments of Nanjing Medical University.

\section{IHC}

IHC was performed as described previously [21]. All the antibodies used are listed in Table S4.

\section{RNA and protein isolation of nuclear and cytoplasmic fractions}

Nuclear and cytoplasmic fractions of CRC cells were separated with a PARIS ${ }^{\mathrm{mm}}$ kit (AM1556; Thermo Fisher Scientific). RNA was isolated according to the protocol and analyzed by qRT-PCR. U6, $18 \mathrm{~S}$, and GAPDH were used as internal controls. The isolated nuclear protein was prepared for the subsequent pulldown assay.

\section{Fluorescence in situ hybridization and immunofluorescence}

Circ-GALNT16-specific Cy3-labeled probe was used to detect the subcellular localization of circ-GALNT16 in DLD-1 and LoVo cells by using a FISH Kit (RiboBio). Briefly, after the cells were fixed with paraformaldehyde for $10 \mathrm{~min}$ and permeabilized for $5 \mathrm{~min}$ by using PBS with $0.5 \%$ Triton X-100, a FISH probe hybridized with a preheated hybridization buffer was mixed with cells and incubated overnight at $37{ }^{\circ} \mathrm{C}$. The cells were then washed with $4 \times$ sodium citrate buffer containing $0.1 \%$ Tween-20 for $5 \mathrm{~min}$ and $1 \times$ SSC for $5 \mathrm{~min}$. The cell nucleus was stained with 4,6-diamidino-2-phenylindole 
A

hsa_circ_0052184 hsa_circ_0124554 hsa_circ_0008234

hsa_circ_0055087 hsa_circ_0044621 hsa_circ_0079875 hsa_circ_0002103

hsa_circ_0122790

hsa_circ_0128882

hsa_circ_0139778

hsa circ 0046136

hsa_circ_0006457

hsa_circ_0138252

hsa_circ_0010620

hsa_circ_0102495

hsa_circ_0031143

hsa_circ_0048545

hsa_circ_0071729

hsa_circ_0029477

hsa_circ_0079250

C
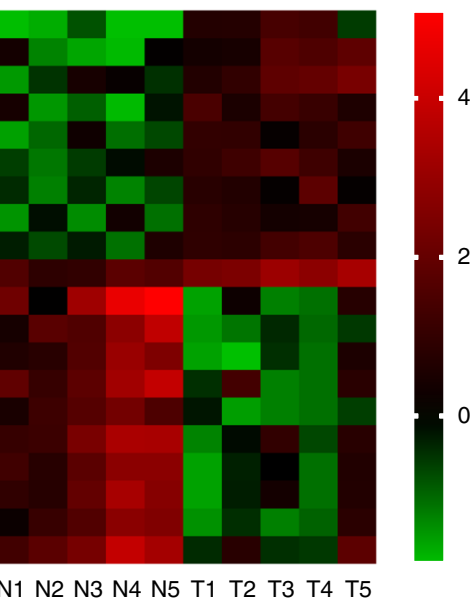

B

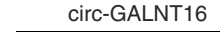

$\frac{\text { GAPDH }}{\longrightarrow>>}$

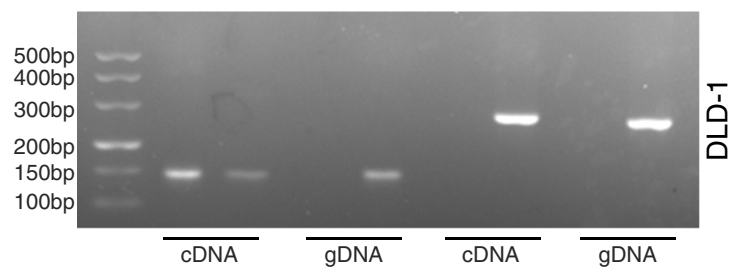

D

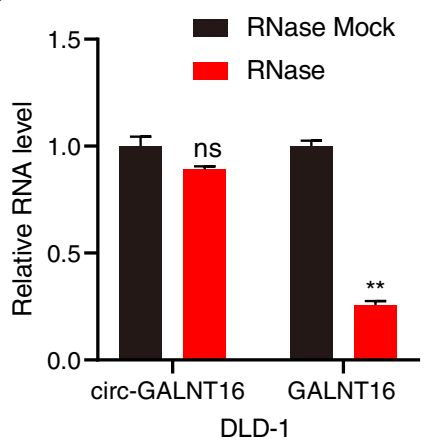

E

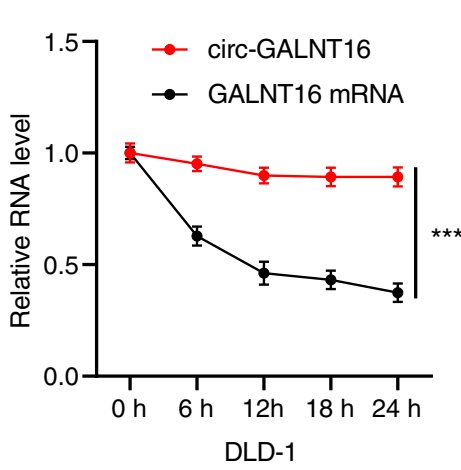

Chr14

GALNT16

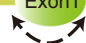

back splicing

$\downarrow$

$290 \mathrm{bp}$

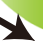

$\Delta \frac{1}{\pi}$
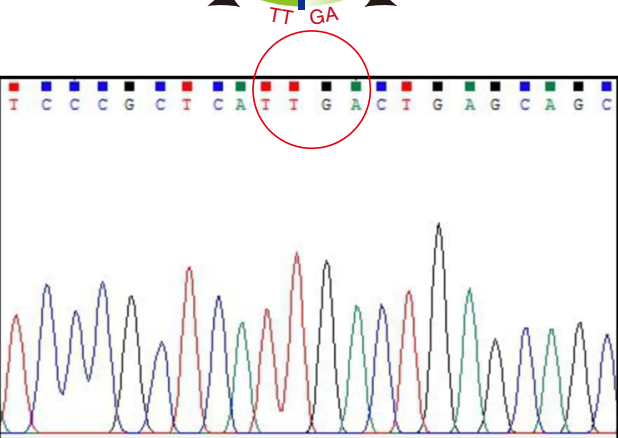

G

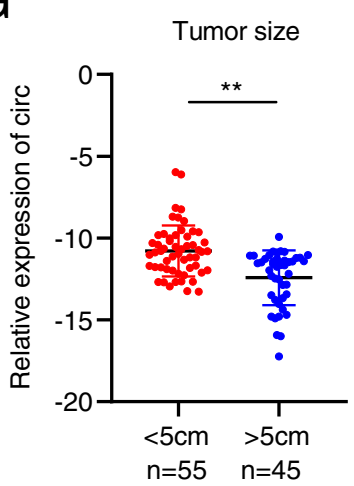

$\mathrm{H}$

F

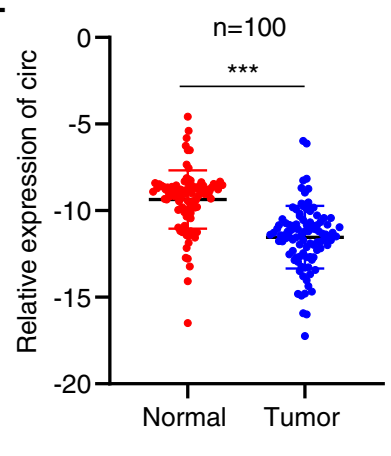
Tumor stage

- circ-GALNT16 low $(\mathrm{n}=50)$

- circ-GALNT16 high $(\mathrm{n}=50)$
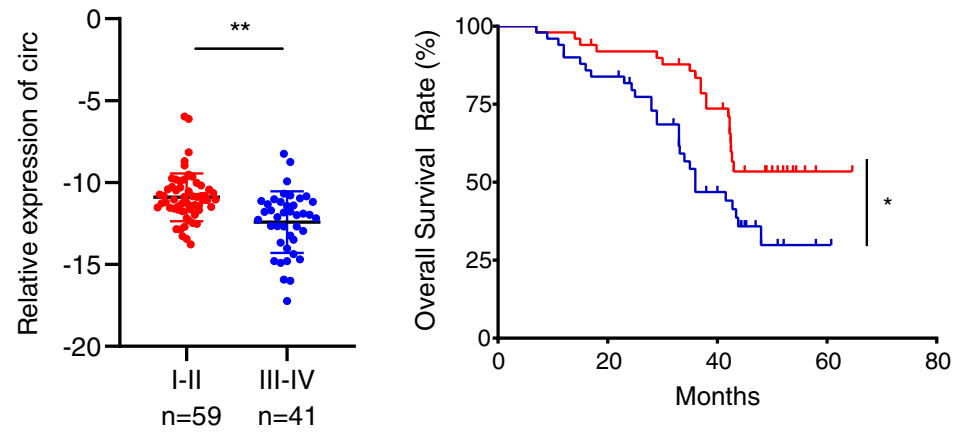

Fig. 1 (See legend on next page.) 
(See figure on previous page.)

Fig. 1 Circ-GALNT16 validation and expression in CRC cells and tissues. a Heatmap of top ten upregulated and downregulated expression circRNAs between CRC tissues and adjacent normal tissues according to the circRNA microarray. $\mathbf{b}$ The schematic illustration showed the back splicing of circ-GALNT16, and sanger sequence validated the splicing site. c PCR and agarose gel electrophoresis confirmed the circular formation of circ-GALNT16, using divergent and convergent primers in gDNA and CDNA of DLD-1. GAPDH was used as a negative control. d, e. Circ-GALN T16 and linear GALNT16 expression levels were detected after RNase R or actinomycin D treatment in DLD-1. f. Relative circ-GALNT16 expression in 100 CRC tissues and matched adjacent normal tissues. g-h. Relative expression of circ-GALNT16 in different tumor size and tumor stage groups. i. Kaplan-Meier plots of the overall survival of CRC patients with high $(n=50)$ and low $(n=50)$ levels of circ-GALNT16. All data are presented as the means \pm SD of three independent experiments. ${ }^{n 5} p>0.05,{ }^{*} p<0.05,{ }^{* *} p<0.01,{ }^{* * *} p<0.001$

(DAPI). The images were obtained using a confocal fluorescence microscope.

For the dual RNA-FISH and immunofluorescence assay, an immunostaining blocking solution (Beyotime) was used for cell blocking for $1 \mathrm{~h}$ after incubation with the FISH probe as described above. The cells were then incubated with hnRNPK antibodies overnight and labeled with fluorescent secondary antibodies for $1 \mathrm{~h}$ in the dark. Finally, DAPI was used for nuclear staining.

\section{RNA pulldown assay and mass spectrometry}

A biotin-labeled pulldown probe targeting circ-GALN T16 and a control probe were designed and synthesized by Ribobio. The RNA pulldown assay was performed using the Pierce Magnetic RNA-Protein Pull-Down Kit according to the protocol (\#20,164; Thermo Fisher Scientific). Mass spectrometry analysis was performed using the elution protein extracted from the RNA pulldown assay. The differential proteins identified by mass spectrometry and the list of RNA-binding proteins (RBPs; http://www.ablife.cc) are shown in Table S5.

\section{Western blotting}

Western blotting (WB) was performed as reported previously [19]. The antibodies are shown in Table S4.

\section{RIP and coimmunoprecipitation assay}

The RNA immunoprecipitation (RIP) assay was performed using a RIP Kit (Millipore, Burlington, MA, USA). In brief, $5 \mu \mathrm{g}$ anti-hnRNPK or anti-FLAG antibodies and magnetic beads were mixed and incubated with cells lysed with RIP lysis buffer supplemented with protease and RNase inhibitors overnight at $4{ }^{\circ} \mathrm{C}$. The immunoprecipitated RNA was obtained for qRT-PCR after digestion with proteinase $\mathrm{K}$ buffer.

Coimmunoprecipitation assay was performed with an IP/Co-IP Kit (\#88,828; Thermo Fisher Scientific) to determine the interactions of hnRNPK with p53 and SENP2. The detailed procedures were performed as previously described [22].

\section{SUMOylation modification analysis and UV irradiation}

The treated cell lysate was diluted 20-fold with a lysis buffer containing $20 \mathrm{mM}$ Tris- $\mathrm{HCl}(\mathrm{pH}=8.0), 150 \mathrm{mM}$
$\mathrm{NaCl}, 2 \mathrm{mM}$ NEM, $1 \times$ protease inhibitor cocktail, and $0.2 \%$ Triton X-100. The lysate was incubated with antihnRNPK antibodies for $4 \mathrm{~h}$ at $4{ }^{\circ} \mathrm{C}$. The lysate was mixed and incubated with protein A/G-Sepharose (sc2003; Santa Cruz Biotechnology, USA) overnight at $4{ }^{\circ} \mathrm{C}$. The resins were washed with a lysis buffer containing $1 \%$ Triton X-100 for three times and boiled in $50 \mu \mathrm{L}$ SDS sample buffer for $10 \mathrm{~min}$. Finally, WB was performed with supernatants using anti-hnRNPK and antiSUMO1 antibodies.

Table 1 Relevance analysis of circ-GALNT16 expression in CRC patients $(n=100)$

\begin{tabular}{|c|c|c|c|c|}
\hline \multirow[t]{2}{*}{ Variables } & \multirow{2}{*}{$\begin{array}{l}\text { All } \\
\text { patients }\end{array}$} & \multicolumn{2}{|c|}{ circ-GALNT16 } & \multirow[t]{2}{*}{$P$ value } \\
\hline & & Low & High & \\
\hline All Cases & 100 & 50 & 50 & \\
\hline \multicolumn{5}{|l|}{ Age (years) } \\
\hline$<60$ & 35 & 16 & 19 & \multirow[t]{2}{*}{0.53} \\
\hline$\geq 60$ & 65 & 34 & 31 & \\
\hline \multicolumn{5}{|l|}{ Gender } \\
\hline Female & 41 & 18 & 23 & \multirow[t]{2}{*}{0.31} \\
\hline Male & 59 & 32 & 27 & \\
\hline \multicolumn{5}{|c|}{ Tumor size (cm) } \\
\hline$<5$ & 55 & 19 & 36 & \multirow[t]{2}{*}{0.001} \\
\hline$\geq 5$ & 45 & 31 & 14 & \\
\hline \multicolumn{5}{|l|}{ Tumor stage } \\
\hline Stage I+II & 59 & 22 & 37 & \multirow[t]{2}{*}{0.002} \\
\hline Stage III+IV & 41 & 28 & 13 & \\
\hline \multicolumn{5}{|c|}{ Lymph node metastasis } \\
\hline No & 61 & 23 & 38 & \multirow[t]{2}{*}{0.002} \\
\hline Yes & 39 & 27 & 12 & \\
\hline \multicolumn{5}{|c|}{ Distant metastasis } \\
\hline No & 93 & 47 & 46 & \multirow[t]{2}{*}{$>0.99$} \\
\hline Yes & 7 & 3 & 4 & \\
\hline \multicolumn{5}{|l|}{ CEA (ng/ml) } \\
\hline$<4.70$ & 46 & 25 & 21 & \multirow[t]{2}{*}{0.42} \\
\hline$\geq 4.70$ & 54 & 25 & 29 & \\
\hline
\end{tabular}

NOTE: CEA carcinoembryonic antigen

All data are presented as the means \pm SD of three independent experiments. $P \leq 0.05$ was considered significant. The bold type represents $P$ values smaller than 0.05 


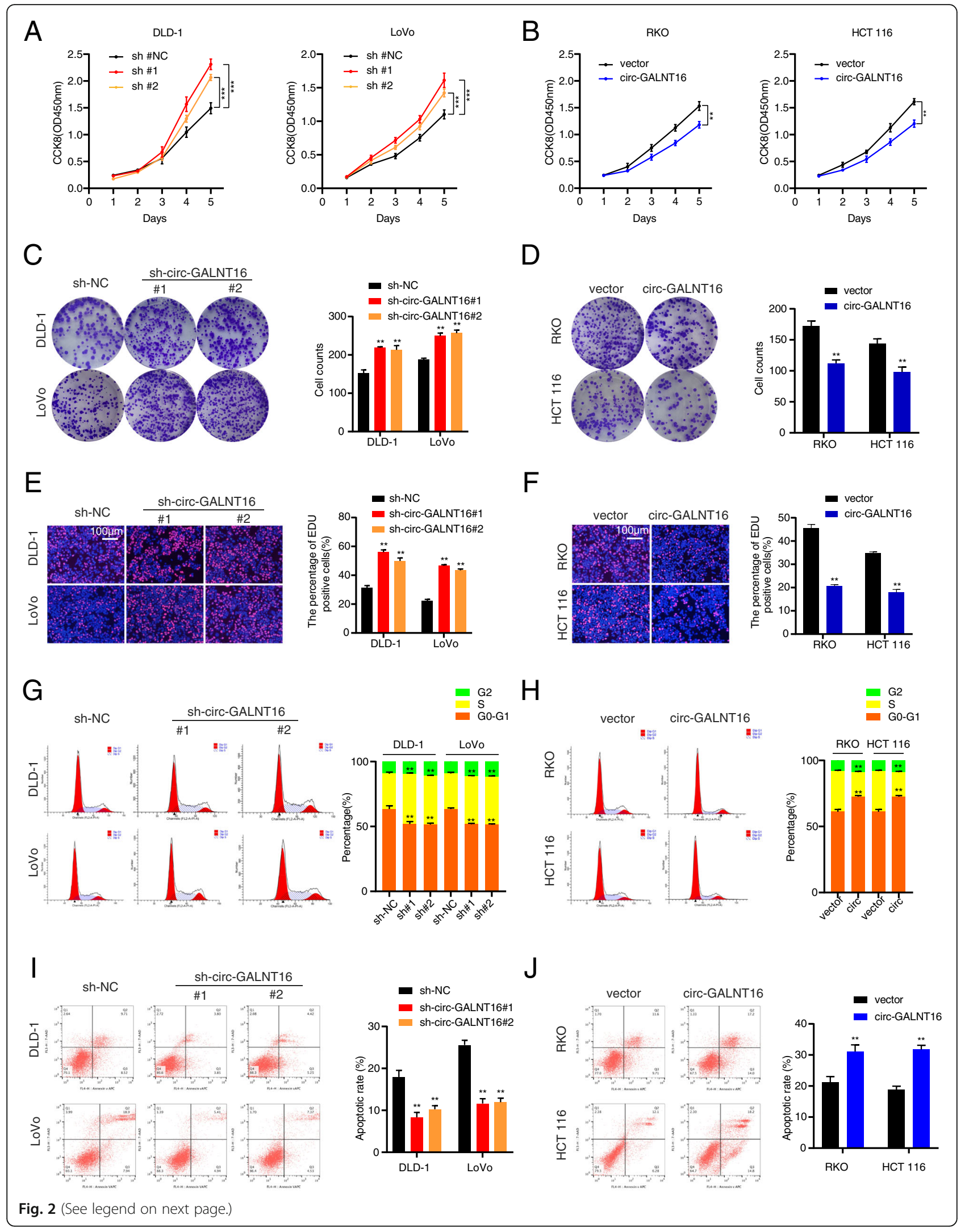


(See figure on previous page.)

Fig. 2 Circ-GALNT16 suppresses the proliferation of CRC cells in vitro. a, b. CCK8 assays were applied to determine the growth curves of circGALNT16 knockdown or overexpression cells. $\mathbf{c}$, d. Colony formation assays were performed to evaluate cell proliferation ability. e, $\mathbf{f}$. EdU assays were performed to assess the cell proliferation ability. $\mathbf{g}, \mathbf{h}$. Cell cycle distributions were detected by flow cytometry in circ-GALNT16 knockdown or overexpression cells. $\mathbf{i}$, j. The apoptotic rates were performed and analyzed after cells were treated with $0.5 \mathrm{mM} \mathrm{H}_{2} \mathrm{O}_{2}$ for $4 \mathrm{~h}$. All data are presented as the means \pm SD of three independent experiments. ${ }^{* *} p<0.01,{ }^{* * *} p<0.001$

The UV treatment was performed as previously reported. Breifly, cells were treated with UV at $50-70 \%$ confluency by UV phototherapy instruments (Sigma, Shanghai, China), whereas irradiation dose $(10 \mathrm{~J} / \mathrm{m} 2)$ was measured with irradiance monitor (Sigma, Shanghai, China). After recovery in medium with $10 \%$ fetal bovine serum for $6 \mathrm{~h}$, cells were harvestd for SUMOylation modification analysis.

\section{RNA sequencing assay}

RNA-seq libraries were prepared after RNA-Seq was performed in RKO cells transfected with a circ-GALN T16 overexpression lentivirus and control cells. First, RNA samples that had passed quality inspection were developed with a starting amount of $1 \mu \mathrm{g}$ and subjected to RNA integrity check by an Agilent 4200 TapeStation. The key steps were as follows: (1) RNA was purified with polyA Oligo magnetic beads; (2) first-strand cDNA synthesis was performed with random hexamer primers; (3) RNA degradation was performed with RNase $\mathrm{H}$, and second-strand cDNA synthesis was carried out using DNA polymerase I; (4) end repair was performed for the double-stranded cDNA fragments, and a single "A" base was added at the 3 -end of each strand; (5) ligation with special sequencing adapters was performed, and PCR amplification was conducted. Finally, a HiSeq 2000 system on Pair End (Illumina, San Diego, CA, USA) was used to sequence the purified cDNA. The mRNA sequencing results are shown in Table S6.

\section{Chromatin immunoprecipitation assays}

A chromatin immunoprecipitation (ChIP) Kit (CST, \#56,383; Danvers, MA, USA) was used for the ChIP assay according to the manufacturer's protocol. Briefly, chromatin fragments were sheared to 200- to 1,000-bp size by using ChIP sonication lysis buffers. The beadsantibody complexes were then incubated with sheared crosslinked chromatin. After the DNA was purified, qRT-PCR was performed using ChIP primers. The sequences of ChIP primer are listed in Table S3.

\section{Statistical analysis}

Each experiment was repeated at least three times. All statistical analyses were performed with GraphPad Prism software (La Jolla, CA, USA) and SPSS 13.0 software (Chicago, IL, USA). The student's $t$-test was performed to analyze the difference between two samples, while
ANOVA was used for tests among more than two groups. Pearson's correlation analysis was performed to estimate the correlation between circ-GALNT16 and Serpine1. The chi-square test was used to analyze the correlation between circ-GALNT16 expression in tumor tissues and relative clinicopathological data. Overall survival (OS) rates were estimated using Kaplan-Meier curves. The significance threshold of each test was set at 0.05 .

\section{Results}

\section{Circ-GALNT16 is downregulated in CRC tissues and} correlates with good prognosis

To determine pivotal circRNAs involved in CRC progression, a circRNA microarray was developed using matched CRC and adjacent normal tissues from 5 patients, and the top 20 upregulated and downregulated circRNAs are shown by heatmap (Fig. 1a). We selected the top 10 differentially expressed circRNAs $(P<0.01)$ for further qRT-PCR verification in 20 paired tumor tissues (Fig. S1a). Circ-GALNT16 (circBase ID: hsa_circ 0102495) was highly downregulated in CRC tissues. Therefore, circ-GALNT16 was chosen for our subsequent in-depth study. Circ-GALNT16 is generated from exon1 of the GALNT16 gene located on chromosome 14 and is 290 nucleotides in length according to the circBase annotation. Sanger sequencing was performed to validate the back-splicing of circ-GALNT16 (Fig. 1b). To confirm the circular formation of circ-GALNT16, both convergent and divergent primers were designed to amplify the linear and back-splicing products. The results demonstrated that the convergent primers for both circ-GALNT16 and GAPDH could amplify products of expected size from both cDNA and genomic DNA (gDNA). Only divergent primers for circ-GALNT16, but not for GAPDH, could amplify a PCR product from cDNA, but not from gDNA (Fig. 1c and Fig. S2a). In addition, qRT-PCR confirmed that circ-GALNT16 was more resistant to RNase $\mathrm{R}$ and actinomycin $\mathrm{D}$ treatment than GALNT16 mRNA in DLD-1 and LoVo cells (Fig. 1d, e and Fig. S2b, c). These results indicated that circ-GALNT16 was a circular, not linear structure. CircGALNT16 was remarkably downregulated in 100 CRC tissues compared to that in paired adjacent normal tissues (Fig. If and Fig. S2d). Correspondingly, the expression of circ-GALNT16 in normal colorectal epithelial cells NCM460 was significantly higher than that in CRC 


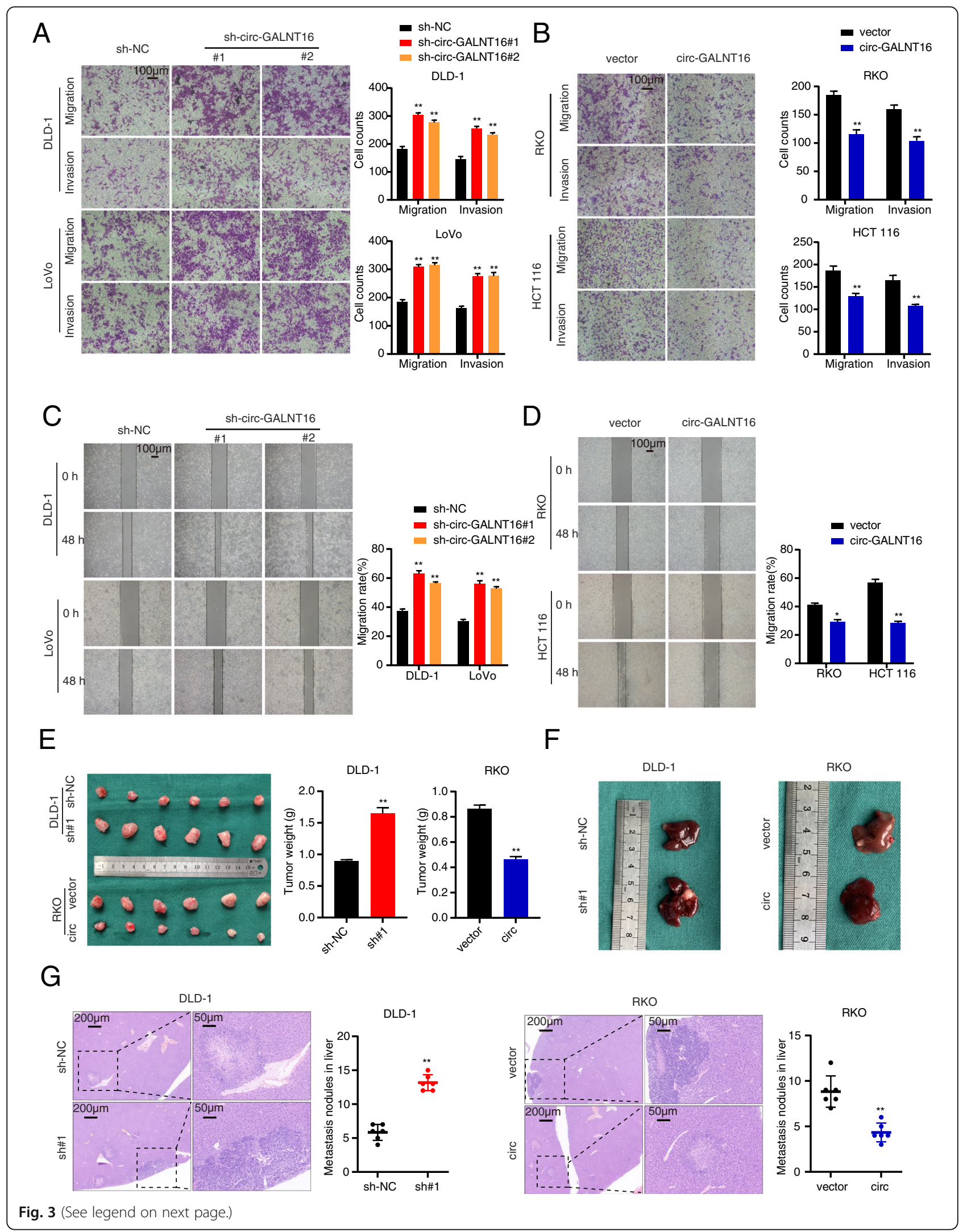


(See figure on previous page.)

Fig. 3 Effects of circ-GALNT16 on the proliferation, migration, and invasion in vitro and vivo. a, b. Transwell assays were applied to evaluate the migration and invasion abilities of CRC cells. c, $\mathbf{d}$. Cell migration ability was assessed by wound healing assay. e. Representative photographs of subcutaneous xenograft tumors were obtained from nude mice, and the tumor weights were measured. $\mathbf{f}, \mathbf{g}$. Representative images and HE staining of liver metastatic tumors. All data are presented as the means \pm SD of three independent experiments. ${ }^{*} p<0.05$, ${ }^{* *} p<0.01$

cell lines (Fig. S2e). The correlation between circ-GALN T16 expression level and clinical subgroups was analyzed. Circ-GALNT16 expression was negatively correlated with tumor size, tumor stage, and lymph node metastasis (Fig. 1g, h and Fig. S2f). To further investigate the link between circ-GALNT16 expression level and clinical characteristics, the specimens were separated into two groups according to the median circ-GALNT16 expression level. As shown in Table 1, significant differences were noted in tumor size, tumor stage, and lymph node metastasis between the high and low circ-GALN T16 expression groups, but there were no differences in sex, age, and carcinoembryonic antigen (CEA) level. In addition, lower circ-GALNT16 expression in tumor tissues was correlated with shorter OS (Fig. 1i). All these clinical data suggested that circ-GALNT16 was downregulated in $\mathrm{CRC}$ and may function as a potential diagnostic and prognostic biomarker for CRC.

\section{Circ-GALNT16 inhibits the proliferation and metastasis of CRC cells in vitro and in vivo}

Because of the comparatively high expression of circGALNT16 in LoVo and DLD-1 cells, three shRNAs against circ-GALNT16 were transfected into LoVo and DLD-1 cells. A circ-GALNT16 overexpression lentivirus was transfected into RKO and HCT 116 cells because of their relatively low expression of circ-GALNT16 in order to elucidate the roles of circ-GALNT16 in CRC proliferation and metastasis. The efficiency of circ-GALNT16 knockdown and overexpression is confirmed by qRTPCR. The expression of GALNT16 mRNA remained unchangeable in circ-GALNT16 knockdown or overexpressing cells (Fig. S3a, b). Both sh-circ-GALNT16\#1 and sh-circ-GALNT16\#2 were chosen for the subsequent cell phenotype assays. CCK-8, colony formation, and EdU assays demonstrated that circ-GALNT16 silencing excessively promoted cell proliferation in LoVo and DLD-1 cells, while circ-GALNT16 overexpression caused a completely opposite phenomenon in RKO and HCT 116 cells (Fig. 2a-f). Flow cytometric assay of cell cycle distribution demonstrated that the knockdown of circ-GALNT16 facilitated the G1 to S transition. However, the overexpression of circ-GALNT16 dramatically increased the number of cells in G0/G1 phase, along with a remarkable decrease in $S$ phase cells (Fig. $2 g$, h). Apoptosis assay showed that the CRC cells transfected with shRNAs had lower apoptotic rates than the control group. Conversely, the apoptotic rates remarkably increased when circ-GALNT16 was overexpressed in RKO and HCT 116 cells (Fig. 2i, j). Additionally, Transwell and scratch wound healing assays confirmed that circ-GALNT16 depletion prominently enhanced the migration and invasion ability of CRC cells, while circGALNT16 overexpression impaired this ability (Fig. 3ad).

DLD-1 cells stably transfected with sh-circ-GALN T16\#1 and RKO cells stably transfected with circ-GALN T16, along with their relative control group, were subcutaneously injected into nude mice to investigate the regulatory effect of circ-GALNT16 on CRC cell proliferation in vivo. The results showed that tumors generated from circ-GALNT16-overexpressing cells had less tumor volume and weight than the control group, while circGALNT16 depletion had an opposite effect (Fig. 3e and Fig. S3c). Furthermore, Ki-67, C-myc, and Serpine1 expression levels were increased in the circ-GALNT16 knockdown group and decreased in the circ-GALNT16 overexpression group as measured by IHC (Fig. S3d). The tumor metastasis assay confirmed that the depletion of circ-GALNT16 resulted in more liver metastatic nodules. In contrast, circ-GALNT16 overexpression caused less liver metastasis than that observed for the control group (Fig. 3f, g). Collectively, all these results showed that circ-GALNT16 inhibited the proliferation and metastasis of CRC cells in vitro and in vivo.

\section{Circ-GALNT16 suppresses the progression of CRC by specifically binding to the KH3 domain of hnRNPK}

To determine the tumor suppression mechanisms of circ-GALNT16 in CRC, we first examined the subcellular location of circ-GALNT16 in CRC cells. Subcellular fractionation assay and fluorescent in situ hybridization (FISH) assay showed that circ-GALNT16 was predominantly located in the nucleus of CRC cells (Fig. 4a, b and Fig. S3e). Next, we performed the biotin-labeled RNA pulldown assay by using a circ-GALNT16-specific probe and a control probe in DLD-1 and LoVo cells, followed by silver staining to identify the potential protein partner of circ-GALNT16 (Fig. 4c). Mass spectrometry revealed that the circ-GALNT16 probe group contained 156 and 96 differential proteins in DLD-1 and LoVo cells, respectively. After overlapping with RBPs, two candidates were selected from the protein partner of circ-GALN T16: heterogeneous nuclear ribonucleoprotein $\mathrm{K}$ (hnRNPK) and FKBP prolyl isomerase 4 (FKBP4). Considering the nuclear location of circ-GALNT16, we 


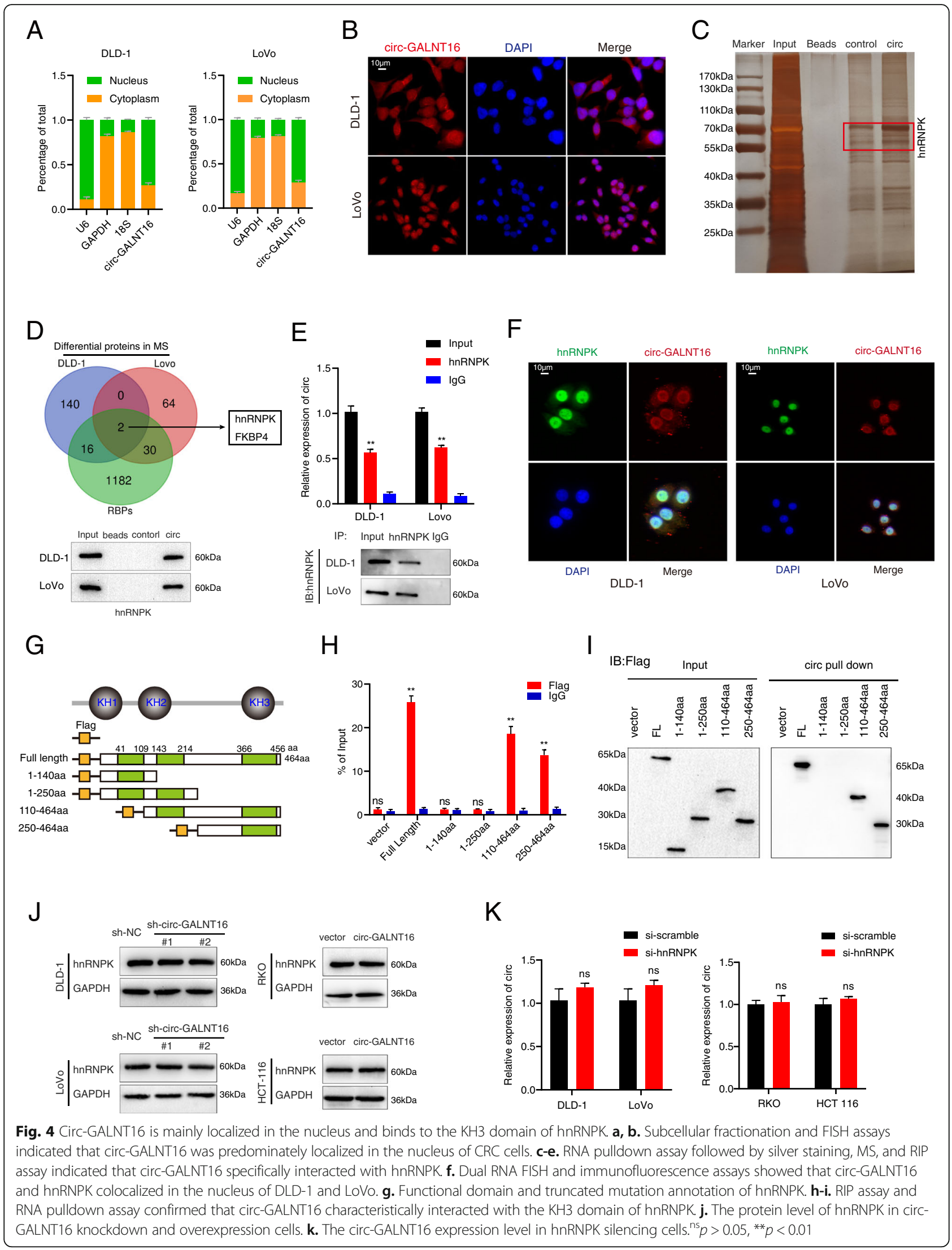




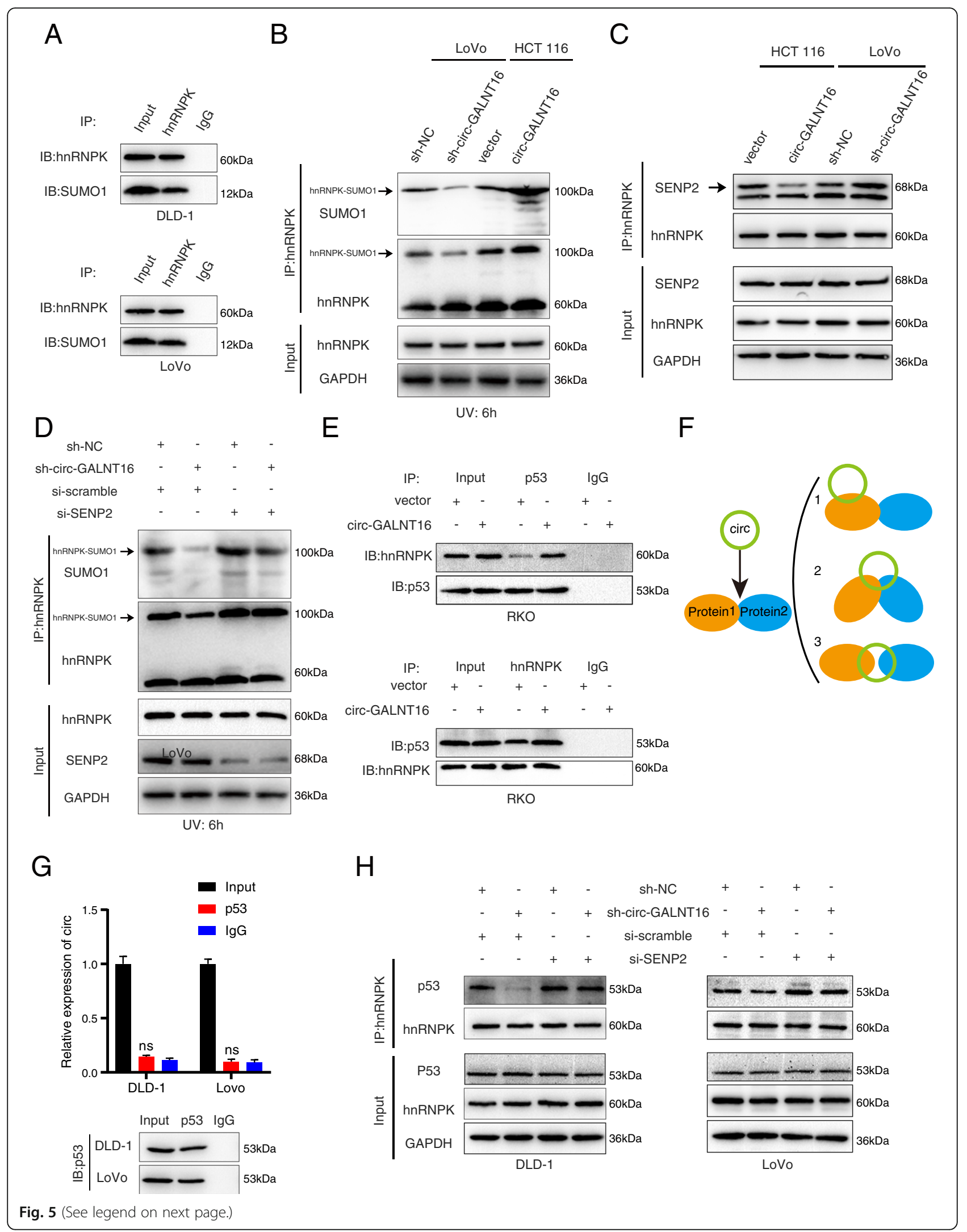


(See figure on previous page.)

Fig. 5 Circ-GALNT16 promotes the interaction between hnRNPK and p53 via inhibiting SENP2-mediated deSUMOylation. a Co-IP assay was performed in protein lysate pulled down by circ-GALNT16 specifical probe using anti-hnRNPK. b SUMOylation modification analysis was performed to identify the levels of hnRNPK SUMOylation in circ-GALNT16 knockdown and overexpression cells at $6 \mathrm{~h}$ after UV stimulation. c The co-IP assay was performed between SENP2 and hnRNPK in circ-GALNT16 silencing and overexpression cells. d SUMOylation modification analysis to explore the levels of hnRNPK SUMOylation in circ-GALNT16 knockdown and SENP2 knockdown cells at $6 \mathrm{~h}$ after UV stimulation. e The co-IP assay elucidated that circ-GALNT16 could mediate the interaction between hnRNPK and p53. $\mathbf{f}$ Three modes of circRNA-protein interactions. $\mathbf{g}$ RIP assay showed p53 did not interact with circ-GALNT16 directly. $\mathbf{h}$ Co-IP assay between p53 and hnRNPK applied in circ-GALNT16 and SENP2 depletion cells. All data are presented as the means \pm SD of three independent experiments. ${ }^{n s} p>0.05,{ }^{* *} p<0.01$

chose hnRNPK as the potential partner of circ-GALN T16, and WB verified the results of mass spectrometry (Fig. 4d and Fig. S4a). The hnRNPK was reported to function as a DNA- and RNA-binding protein and to regulate a large number of biological processes and cancer pathogenesis [13]. Additionally, previous studies showed that hnRNPK could influence CRC progression by interacting with noncoding RNA [23, 24]. Next, hnRNPK silencing cells were constructed (Fig. S4b). The RNA pulldown assay was then performed again with the nucleoprotein of DLD-1 cells (Fig. S4c). Additionally, the RIP assay showed that hnRNPK could specifically enrich circ-GALNT16 (Fig. 4e). Dual RNA-FISH and immunofluorescence assay showed the co-localization of circGALNT16 and hnRNPK (Fig. 4f). We designed four truncated hnRNPK plasmids aiming at three $\mathrm{K}$ homology $(\mathrm{KH})$ domains that mediated nucleic acid binding to determine which domain circ-GALNT16 interacted with. Protein domain mapping and RIP assay showed that circGALNT16 interacted with the KH3 domain of hnRNPK (Fig. 4g-i). CCK8 and Transwell assays also indicated that circ-GALNT16 depletion could not affect the proliferation and metastatic ability of CRC cells while hnRNPK was knocked down (Fig. S4d, e). The mRNA and protein levels of hnRNPK did not alter in circ-GALNT16 knockdown or overexpressing cells (Fig. $4 \mathrm{j}$ and Fig. S4f). The expression of circ-GALNT16 also remained intact while hnRNPK was knocked-down (Fig. 4k). Collectively, circ-GALNT16 restrained $\mathrm{CRC}$ progression by interacting with the $\mathrm{KH} 3$ domain of hnRNPK.

\section{Circ-GALNT16 enhances hnRNPK-p53 interaction by inhibiting SENP2-mediated hnRNPK deSUMOylation, and hnRNPK facilitates the nuclear accumulation of circ-GALN T16}

Given that circ-GALNT16 specifically interacts with hnRNPK, we next investigated how circ-GALNT16 restrained CRC progression through hnRNPK. Because the protein levels of hnRNPK were not changed in circGALNT16 knockdown or overexpressing cells, we assumed that circ-GALNT16 could influence some kind of posttranslational modification of hnRNPK by binding to it. Previous studies have reported that the KH3 domain was the site of hnRNPK SUMOylation and that noncoding RNA could regulate the SUMOylation of hnRNPK in hepatocellular carcinoma. SUMOylation is a vital posttranslational modification of hnRNPK involved in transcriptional regulation, DNA repair, etc. [14, 25]. Therefore, we wondered whether circ-GALNT16 could regulate the SUMOylation modification of hnRNPK. A co-IP assay was performed with the protein lysate pulled down by the circ-GALNT16-specific probe, and the results indicated that circ-GALNT16 might mediate the interaction between hnRNPK and SUMO1 (Fig. 5a). Next, SUMOylation modification analysis was performed. The results showed that knockdown of circGALNT16 could decrease the SUMOylation modification of hnRNPK at $100 \mathrm{kDa}$, which was the theoretical molecular weight of hnRNPK conjugated with SUMO1 (Fig. S5a). SUMOylation is easily lost after lysis due to the high activity of SUMO isopeptidase, which may lead to the expression level of SUMOylation of hnRNPK seems little [26]. It has been reported that ultraviolet exposure could accumulate the SUMOylation of hnRNPK and impair the interaction between hnRNPK and SUMO-specific peptidase 2 (SENP2) at $6 \mathrm{~h}$ after UV stimulation [16]. Cells were harvested at $6 \mathrm{~h}$ after UV exposure for SUMOylation modification analysis, and the results showed that circ-GALNT16 could enhance the SUMOylation modification of hnRNPK with UV stimulation (Fig. 5b). SENP2 can antagonistically regulate hnRNPK SUMOylation in HeLa and HCC cells [16, 25]. However, it remains unclear whether SENP2 could facilitate the deSUMOylation of hnRNPK in CRC cells. A co-IP assay confirmed that SENP2 could bind to hnRNPK in CRC cells (Fig. S5b). We then found that circ-GALNT16 overexpression could impair the interaction between SENP2 and hnRNPK, and circ-GALN T16 knockdown enhanced this interaction (Fig. 5c). Similar results were presented in cells with UV exposure (Fig. S5c). SENP2 knockdown cells were constructed (Fig. S5d). SUMOylation modification analysis revealed that circ-GALNT16 enhanced the SUMOylation of hnRNPK through SENP2 (Fig. 5d and Fig. S5e). These data indicated that circ-GALNT16 could weaken the deSUMOylation modification of hnRNPK through binding to hnRNPK and blocking the interaction between hnRNPK and SENP2. 


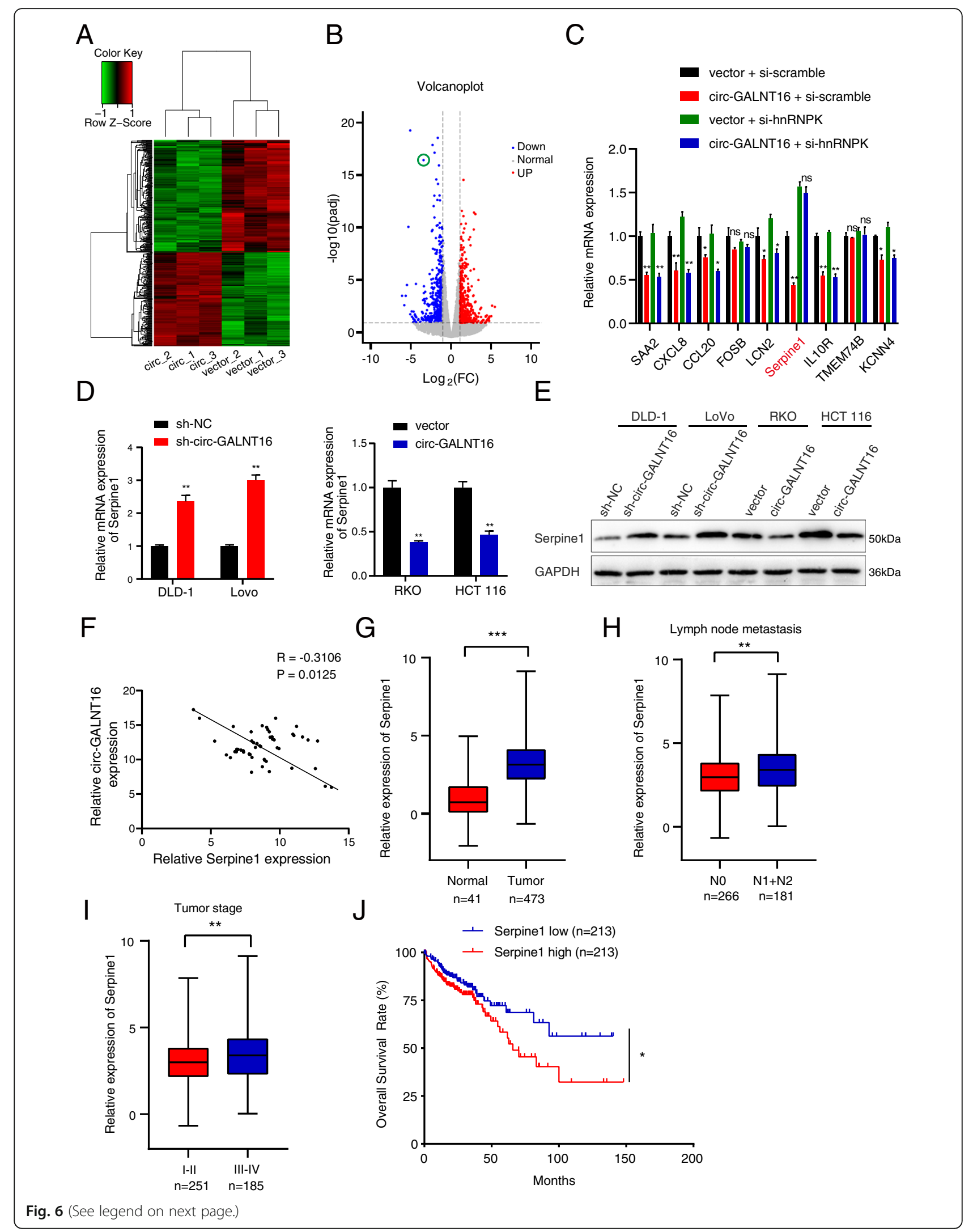


(See figure on previous page.)

Fig. 6 Serpine1 is downregulated by circ-GALNT16 and has a connection with CRC progression. a, b. Heatmap and scatter diagram showed the RNA-seq result in circ-GALNT16 overexpression and control RKO cells. c. Several candidates were validated by qRT-PCR. d, e. The mRNA and protein levels of Serpine1 in circ-GALNT16 knockdown and overexpression cells. f. Correlation analysis between circ-GALNT16 and Serpine1. $(R=$ $-0.3106, P=0.0125) \mathbf{g}-\mathbf{j}$. Serpine1 expression in CRC and the correlation with relative clinical features analysis from TCGA. All data are presented as the means \pm SD of three independent experiments. ${ }^{n s} p>0.05,{ }^{*} p<0.05,{ }^{* *} p<0.01,{ }^{* * *} p<0.001$

Several studies have reported that the SUMOylation of hnRNPK could enhance the interaction between hnRNPK and $\mathrm{p} 53$, which plays a major role in cancer progression $[16,17,25]$. A co-IP assay indicated that circ-GALNT16 could enhance the interaction between hnRNPK and p53 (Fig. 5e). A previous review summarized three approaches through which circRNAs regulate interactions of two proteins: (1) regulating the function of one protein but not binding to the other protein; (2) forming a scaffold and cementing their interaction by binding to both of them; and (3) acting as a dissociator between two proteins (Fig. 5f) [27]. Because circ-GALNT16 did not physically bind to p53, we hypothesized that circ-GALNT16 regulated the interaction between hnRNPK and p53 by enhancing the SUMOylation of hnRNPK, rather than acting as a scaffold between hnRNPK and p53 (Fig. 5g and Fig. S5f). SENP2 silencing could abolish the dissociation between hnRNPK and p53 and inhibit the proliferation of CRC cells caused by knockdown of circ-GALNT16 (Fig. 5h, and Fig. S5g). Collectively, circ-GALNT16 enhanced the interaction between hnRNPK and p53 by blocking SENP2-mediated deSUMOylation of hnRNPK.

After we proved that circ-GALNT16 could specifically interact with hnRNPK and hnRNPK was reported to play a pivotal role in RNA transcription and alternative splicing [28-30], we wondered whether hnRNPK could regulate the transcription or splicing of circ-GALNT16. Neither circ-GALNT16 nor GALNT16 showed a significant variation in their expression while hnRNPK was knocked down, indicating that hnRNPK did not participate in the transcription or splicing of circ-GALNT16 (Fig. S6a). A previous study reported that hnRNPK played an important role in driving long noncoding RNA nuclear enrichment [31]. Considering that most circular RNAs are mainly localized in the cytoplasm and function as miRNA sponge, but circ-GALNT16 had nuclear localization, we again performed RNA FISH and subcellular fractionation assay in hnRNPK-depleted cells; the results showed that circ-GALNT16 tended to accumulate in the cytoplasm of cells with low hnRNPK expression (Fig. S6b, c). These results indicated hnRNPK might contribute to the nuclear localization of circ-GALNT16.

\section{Circ-GALNT16 impairs the expression of Serpine1 in CRC}

Given that circ-GALNT16 could enhance the level of the hnRNPK-p53 transcriptional complex, RNA-seq was performed in circ-GALNT16-overexpressing and related control
RKO cells to determine how circ-GALNT16 regulated CRC progression at the transcriptional level (Fig. 6a). A total of 265 genes were downregulated, and 221 genes were upregulated (Fig. 6b). The expression analysis of the top 30 differential genes in CRC tissues was performed using the public GEPIA dataset based on the TCGA (http://gepia.cancer-pku.cn/index. $\mathrm{html})$. Nine genes were selected as target candidates of circGALNT16. The results of qRT-PCR showed that only Serpine1 could be downregulated by circ-GALNT16 overexpression but exhibited no significant difference when hnRNPK was silenced (Fig. 6c). Coincidentally, published reports and the KEGG (Kyoto Encyclopedia of Genes and Genomes) pathway database indicated that Serpine1 could be regulated by p53 [32]. The mRNA and protein levels of Serpine1 in circ-GALNT16 knockdown and overexpressing cells revealed that Serpine1 could be antagonistically regulated by circGALNT16 (Fig. 6d, e). The expression of Serpine1 in 52 tumor tissues was detected, and the results showed that Serpine1 mRNA level was negatively correlated by circ-GALN T16 expression (Fig. 6f). We then analyzed the correlation between Serpine1 expression and relative clinical features from TCGA. Serpine1 was upregulated in CRC tissues and positively correlated with advanced tumor stage, lymphoid metastasis, and lower OS rate (Fig. 6g-j). These features were contrary to the characteristics of circ-GALNT16.

\section{Circ-GALNT16 suppresses the proliferation and metastasis} of CRC by downregulating Serpine1

Given that circ-GALNT16 could suppress the proliferation and metastasis of CRC and the expression of Serpine1, we hypothesized that circ-GALNT16 might suppress CRC progression by inhibiting Serpine1 expression. First, Serpine1-specific siRNA was transfected into DLD-1 and LoVo cells, and an overexpression plasmid was transfected into RKO and HCT 116 cells (Fig. S7a). Cell proliferation assays showed that Serpine1 silencing could remarkably decrease the proliferation ability of circ-GALNT16 knockdown cells. In contrast, Serpine1 overexpression could promote the proliferation ability of circ-GALNT16-overexpressing cells (Fig. S7b-g). Besides, Cell cycle assays showed that the knockdown of circGALNT16 decreased the number of cells in G0/G1 phase along with a remarkable increase in $S$ phase cells, and this effect could be rescued by the knockdown of Serpine1 (Fig. 7a). The overexpression of circ-GALNT16 could impair the G1 to S translation, and Serpine1 


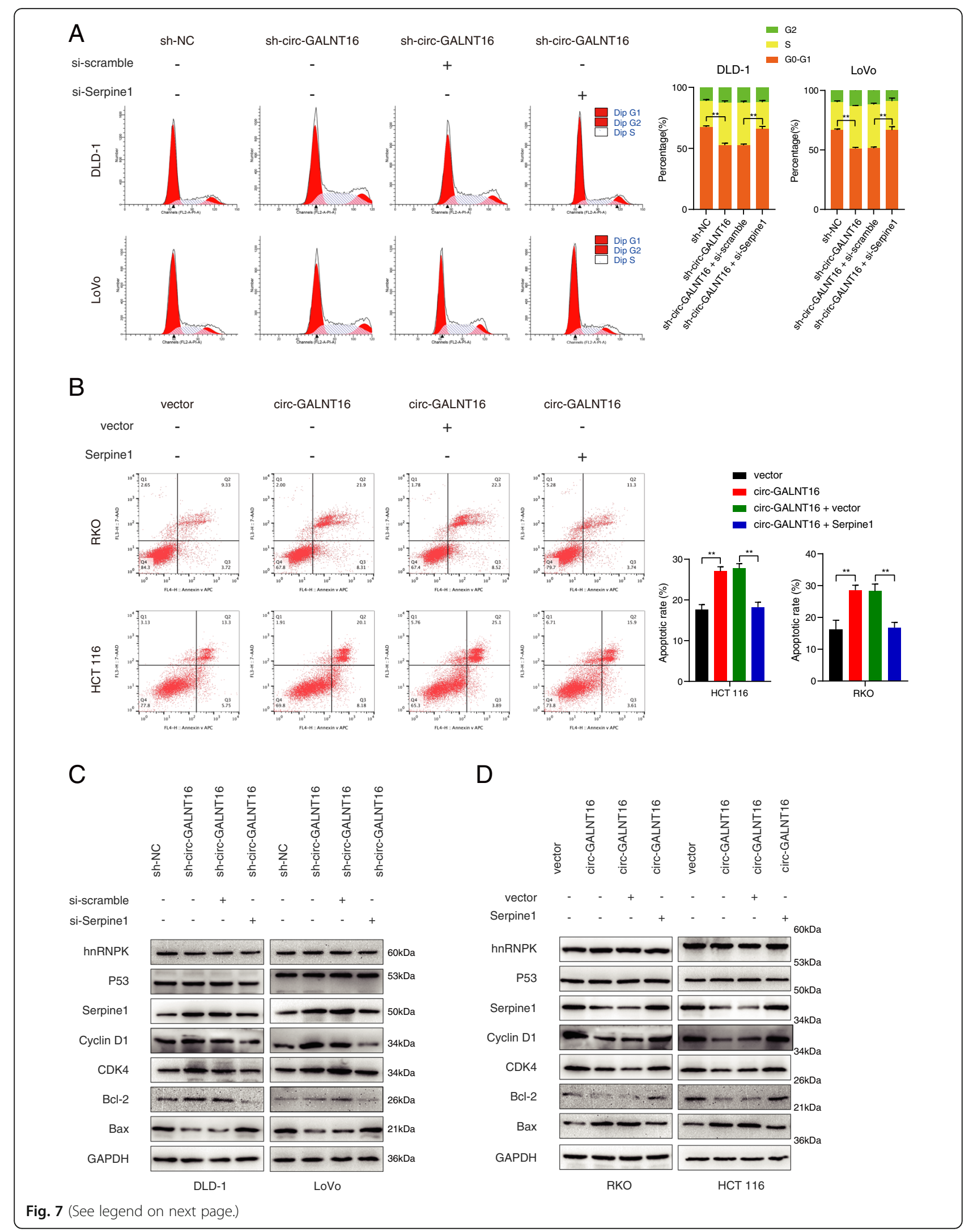


(See figure on previous page.)

Fig. 7 Circ-GALNT16 regulates cell cycle and apoptosis through Serpine1. a Cell cycle assays were performed in sh-circ-GALNT16 and si-Serpine1 co-transfected cells. $\mathbf{b}$ Cell apoptosis assays were performed in circ-GALNT16 overexpression and Serpine1 overexpression groups. c-d. The expression of cell cycle and apoptosis makers (Cyclin D1, CDK4, BCl-2, and Bax) along with hnRNPK, p53, and Serpine1 were detected by western blot in relatively treated cells. ${ }^{* *} p<0.01$

overexpression could enhance the translation (Fig. S8a). Cell apoptosis assay demonstrated that the proapoptotic effect of circ-GALNT16 could be weakened by Serpine1(Fig. $7 \mathrm{~b}$ and Fig. S8b). It has been reported that cyclin D/ CDK4 induced G1/S transition, while Bcl-2/Bax regulated cell apoptosis antagonistically [33, 34]. The expression of CDK4, Cyclin D1, Bcl-2, and Bax were determined, and results were consistent with previous cell cycle and apoptosis assays (Fig. 7c, d). Wound healing assay and transwell assays showed that circ-GALNT16 could suppress the metastasis ability of CRC through Serpine1 (Fig. S9a-d). Taken together, these results showed that circ-GALNT16 suppresses the proliferation and metastasis of CRC by downregulating Serpine1.

\section{Circ-GALNT16 attenuates Serpine1 and enhances the p21 mRNA expression level by regulating the sequence- specific DNA-binding ability of the hnRNPK-p53 complex} Given that circ-GALNT16 could enhance the hnRNPKp53 complex and that Serpine1 was a p53 target gene, we hypothesized that circ-GALNT16 might regulate Serpine1 by enhancing the level of the hnRNPK-p53 complex. First, we observed that the inhibition of Serpine1 caused by circ-GALNT16 could be abolished by hnRNPK silencing (Fig. 8a, b). Moreover, considering that a previous study reported that SUMOylation of hnRNPK could regulate the sequence-specific DNA-binding ability of p53 and exert different effects on the individual p53 target gene, the expression of $\mathrm{p} 21$, a representative p53 target antioncogene, was detected together with Serpine1. P21 was downregulated by circ-GALNT16 knockdown and upregulated by circ-GALNT16 overexpression, but it showed no remarkable divergence during simultaneous hnRNPK knockdown (Fig. S10a, b). Additionally, the expression of Serpine1 showed no significant difference in circ-GALN T16-depleted cells when SENP2 was silenced, indicating that the SUMOylation of hnRNPK played a vital role in Serpine1 regulation caused by circ-GALNT16 (Fig. 8c). The inhibitory effect of circ-GALNT16 knockdown on p21 mRNA level was abolished by SENP2 silencing (Fig. S10c). Pifithrin- $\alpha$, a specific inhibitor of p53 transcriptional activity, could rescue the promotion of Serpine1 expression caused by circ-GALNT16 knockdown (Fig. 8d). Pifithrin- $\alpha$ could also abolish the promotion effect of circGALNT16 overexpression on the p21 mRNA level (Fig. S10d). ChIP assays revealed that circ-GALNT16 depletion enhanced the binding of hnRNPK and p53 to the promoter region of Serpine1 and decreased the binding to the promoter region of $\mathrm{p} 21$; these findings were consistent with previous results. Circ-GALNT16 overexpression had a contrary effect (Fig. 8e, f and Fig. S10e, f). All these results indicated that circ-GALNT16 regulated Serpine1 and p21 expression by modulating the sequence-specific DNA-binding ability of the SENP2-mediated hnRNPKp53 complex.

\section{Discussion}

Several studies have indicated that circRNAs, a group of novel noncoding RNAs, can regulate cancer progression through multiple approaches such as functioning as miRNA sponges and interacting with proteins, and thus, they have the potential to function as critical clinical diagnostic and prognostic biomarkers for malignant tumors [35-38]. In the present study, we investigated the role of a novel circRNA, circ-GALNT16, as a tumor suppressor in CRC. Circ-GALNT16 was found to be downregulated in CRC tissues and cells. The low expression of circ-GALNT16 predicted advanced tumor size, tumor stage, lymphatic metastasis, and poor prognosis. Our phenotype experiment and experiments in mice models revealed that circ-GALNT16 suppressed the proliferation, migration, and invasion ability of CRC cell lines. In particular, circ-GALNT16 enhanced hnRNPK-p53 interaction by inhibiting SENP2-mediated deSUMOylation of hnRNPK, which regulated the sequence-specific DNAbinding ability of the hnRNPK-p53 complex (Fig. 9).

Recent studies have shown that circRNAs function as competing endogenous RNAs (ceRNAs) of miRNAs in the cytoplasm $[39,40]$. We accidentally found that circGALNT16 was predominantly located in the nucleus of CRC cells, thus excluding the possibility of it being a miRNA sponge. The mechanisms of circRNAs with nuclear localization in tumor progression remain obscure and poorly investigated, especially in CRC. In the present study, we found that circ-GALNT16 could specifically bind to the KH3 domain of hnRNPK, which is a multifunctional protein involved in transcriptional regulation, RNA splicing, RNA translocation, and chromatin remodeling [13, 28, 31]. We also found that circ-GALN T16 could enhance the interaction between hnRNPK and p53 by inhibiting SENP2-mediated deSUMOylation of hnRNPK. RNA-seq and qRT-PCR validation indicated that circ-GALNT16 could downregulate the oncogene Serpine1 and upregulate the antioncogene $\mathrm{p} 21$ by regulating the sequence-specific DNA-binding ability of the hnRNPK-p53 transcriptional complex. Collectively, our 

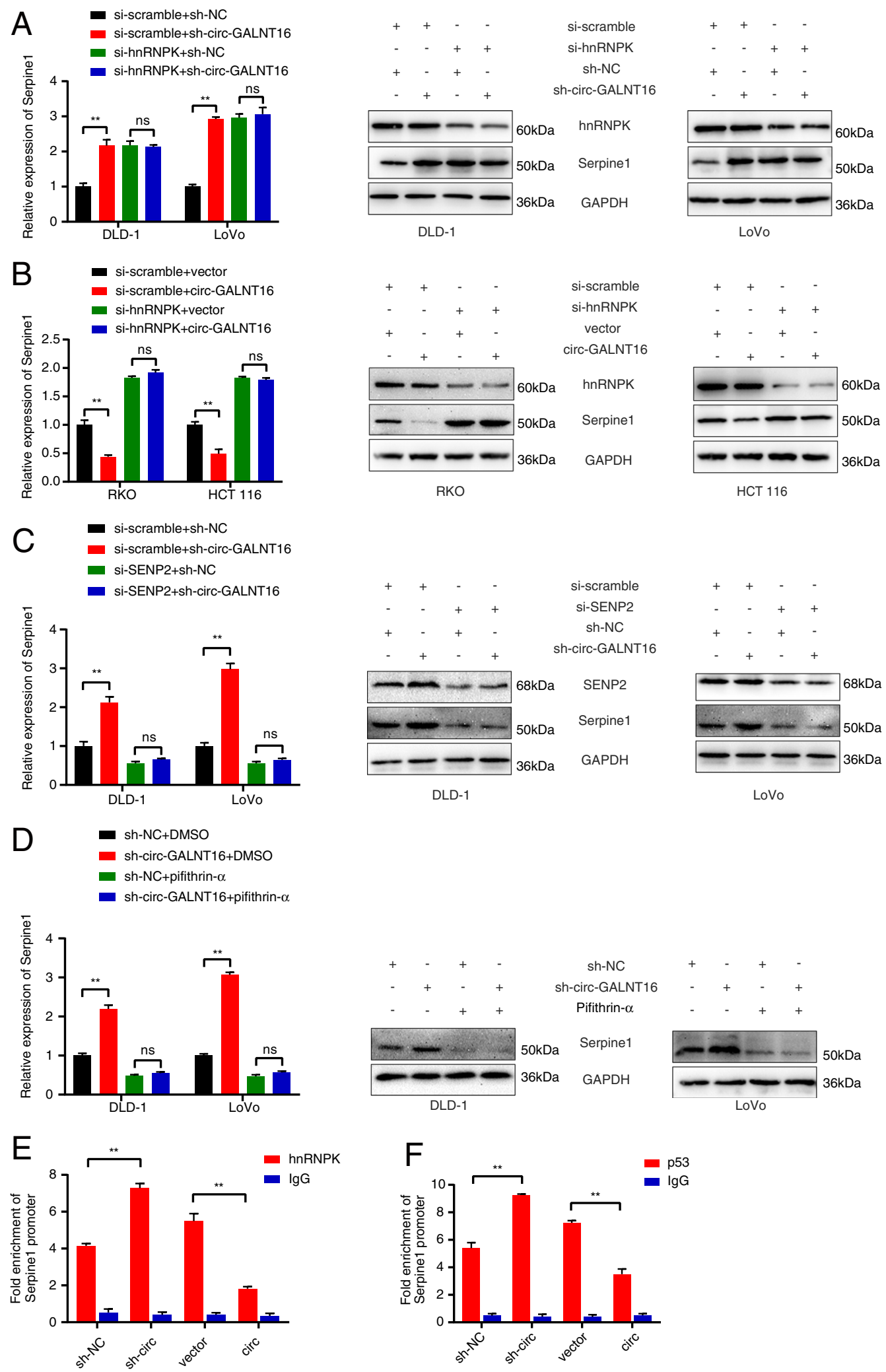

Fig. 8 (See legend on next page.) 
(See figure on previous page.)

Fig. 8 Circ-GALNT16 regulates Serpine1 through the SUMOylation of hnRNPK and p53. a, b. The mRNA and protein level of Serpine1 was detected in circ-GALNT16 knockdown and overexpression cells while hnRNPK was silenced. c. The expression of Serpine1 was detected in circGALNT16 knockdown cells while SENP2 was silenced. d. The expression of Serpine1 in circ-GALNT16 silencing cells was detected with the

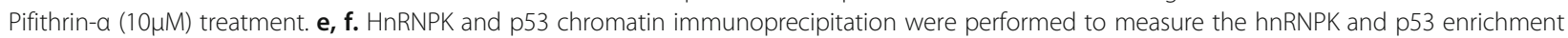
at the promoter region(s) of Serpine1 in DLD-1 and RKO. All data are presented as the means \pm SD of three independent experiments.

${ }^{n s} p>0.05,{ }^{* *} p<0.01$

study showed that the circ-GALNT16/hnRNPK/p53 axis could suppress Serpine1 expression in CRC.

Many studies have demonstrated that various posttranslational modifications of hnRNPK, including phosphorylation, ubiquitination, methylation, and SUMOylation, play critical roles in hnRNPK function coactivation [14]. Although several circRNAs have been reported to interact with hnRNPK, the circRNA that regulates the posttranslational modification of hnRNPK has not yet been detected [23, 24, 41]. Moreover, the role of circRNA in the SUMOylation of proteins has not yet been elucidated. In our present study, we first identified circ-GALNT16 that could specifically interact with the KH3 domain of hnRNPK and enhance its SUMOylation modification by inhibiting SENP2 binding to hnRNPK. These results indicated that circRNA could regulate the SUMOylation modification of proteins and provided a new insight for circRNA-based targeting therapy.

RNA-seq and the subsequent qRT-PCR verified that Serpine1 could be downregulated by circ-GALNT16 through the hnRNPK-p53 complex. Several studies have reported that Serpine1 is elevated in multiple tumor tissues, including colorectal cancer, and correlates with poor outcomes. Both p53 and NF- $\mathrm{kB}$ have been reported to participate in Serpine1 transcriptional regulation [4244]. However, the particular mechanisms of Serpine1 remain unclear, especially in CRC. A previous study showed that SUMOylation of hnRNPK induced different effects on the p53 target gene by regulating the sequence-specific DNA-binding ability of p53 [25]. Therefore, after we found that the inhibition of hnRNPK deSUMOylation caused by circ-GALNT16 overexpression could inhibit Serpine1 expression, we proved that

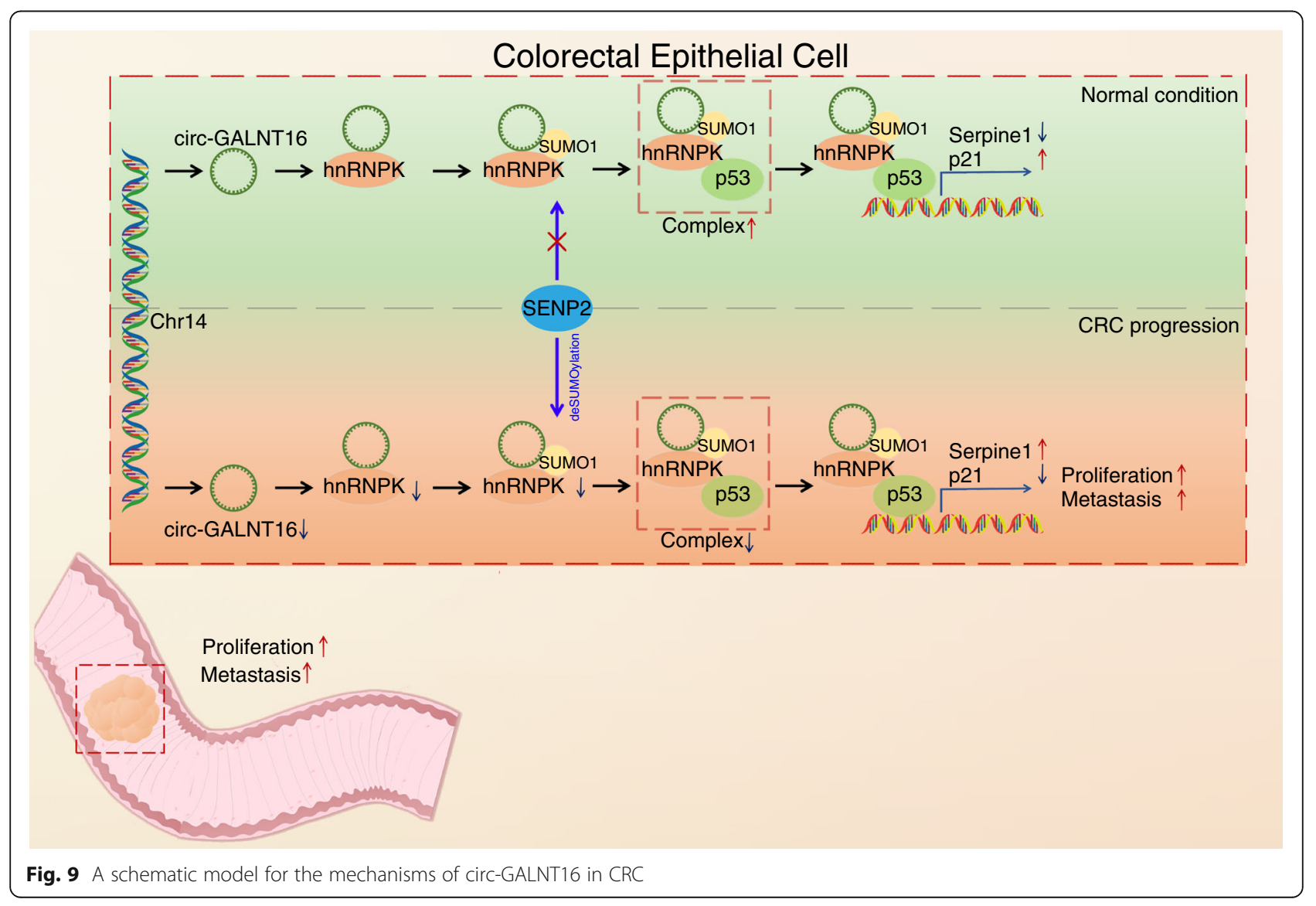


circ-GALNT16 overexpression could upregulate the p21 mRNA level through the hnRNPK-p53 complex; this finding was consistent with a previous study [25]. Intriguingly, ChIP assay showed that circ-GALNT16 could attenuate the enrichment of hnRNPK and p53 at Serpine1 promoters but facilitated the enrichment at p21 promoters. The underlying mechanisms for this phenomenon deserve further investigation.

To investigate how circ-GALNT16 mediated the SUMOylation of hnRNPK, we identified SENP2, which was reported to antagonistically mediate the SUMOylation of hnRNPK in HeLa and HCC cells and could interact with hnRNPK in CRC cells $[16,25]$. The overexpression of circ-GALNT16 could attenuate the interaction between hnRNPK and SENP2, while circGALNT16 knockdown enhanced their interaction. However, we did not determine how circ-GALNT16 mediated the interaction between SENP2 and hnRNPK and the SUMOylation of hnRNPK by specifically binding to the KH3 domain of hnRNPK. A previous study showed that PSTAR could also inhibit the SENP2-mediated deSUMOylation of hnRNPK by interacting with the KH3 domain, which is consistent with our result [25]. Hence, we believe that some correlation might exist between them.

In the present study, we revealed the tumor suppression effect and feasible molecular mechanisms of the novel circRNA circ-GALNT16 in CRC. Circ-GALNT16 was highly downregulated in CRC tissues, which indicated that it might serve as a diagnostic and prognostic biomarker. Our mice models showed that circ-GALN T16 overexpression could inhibit the proliferation and metastatic ability of CRC in vivo, thus indicating that circ-GALNT16 might have the potential to act as a therapeutic target for CRC.

\section{Conclusions}

In summary, we identified a novel circRNA, named circ-GALNT16, that was downregulated in CRC and was negatively associated with a poor prognosis. Circ-GALNT16 could suppress the proliferation and aggressiveness of CRC cells and attenuate Serpine1 expression by inhibiting SENP2-mediated hnRNPK deSUMOylation and modulating the sequencespecific DNA-binding ability of the hnRNPK-p53 complex.

\footnotetext{
Abbreviations

CRC: Colorectal cancer; circRNA: Circular RNA; GALNT16: Polypeptide Nacetylgalactosaminyltransferase 16; hnRNPK: Heterogeneous nuclear ribonucleoprotein K; SUMO1: Small ubiquitin-related modifier 1; OS: Overall survival rate; GAPDH: Glyceraldehyde 3-phosphate dehydrogenase; SENP2: SUMO specific peptidase 2; Serpine1: Serpin family E member 1; EDU: 5-Ethynyl-2'-deoxyuridine; CCK8: Cell Counting Kit-8; IHC: Immunohistochemistry; qRT-PCR: Quantitative reverse transcription polymerase chain reaction; FISH: Fluorescence in situ hybridization; MS: Mass
}

spectrometry; RBPs: RNA binding proteins; NEM: N-ethylmaleimide; shRNA: :Short hairpin RNA; HCC: Hepatocellular carcinoma; FKBP4: FKBP prolyl isomerase 4; UV: Ultraviolet

\section{Supplementary Information}

The online version contains supplementary material available at https://doi. org/10.1186/s13046-021-02074-7.

Additional file 1: Figure S1. The screening of circRNAs. a. The expression of top5 upregulated and downregulated circRNAs in 20 pairs of CRC tissues and relative adjacent normal tissues. All data are presented as the means \pm SD of three independent experiments. ${ }^{n s} p>0.05,{ }^{*} p<$ $0.05,{ }^{* *} p<0.01,{ }^{* * *} p<0.001$

Additional file 2: Figure S2. Circ-GALNT16 validation and expression in CRC tissues and cells. a. PCR and agarose gel electrophoresis confirmed the circular formation of circ-GALNT16, using divergent and convergent primers in gDNA and cDNA of LoVo. b, c. The expression of circ-GALNT16 and linear GALNT16 was detected after RNase R or actinomycin D treatment in LoVo. d. Relative expression of circ-GALNT16 in 100 pairs of CRC and adjacent normal tissues. e. Relative expression of circ-GALNT16 in CRC cell lines and normal epithelial colon cell NCM460. f. Relative expression of circ-GALNT16 in tissue groups with or without lymph node metastasis. All data are presented as the means \pm SD of three independent experiments. ${ }^{\mathrm{ns}} p>0.05{ }^{* *} p<0.01,{ }^{* * *} p<0.001$.

Additional file 3: Figure S3. Circ-GALNT16 stably knockdown and overexpression efficiency, and phenotype assays in vitro and vivo. a. The efficiency of circ-GALNT16 knockdown in DLD-1 and LoVo. The expression of GALNT16 mRNA remained unchangeable. b. The efficiency of circGALNT16 overexpression in RKO and HCT 116. The expression of GALN T16 mRNA level was detected at the same time. $c$. The tumor volumes were measured every 5 days since the subcutaneous tumors were macroscopic. d. Protein levels of Ki67, C-myc, and Serpine1 in the tumor samples were measured by IHC. e. Subcellular fractionation indicated that circ-GALNT16 was predominately localized in the nucleus of CRC cells. All data are presented as the means \pm SD of three independent experiments. ${ }^{n s} p>0.05^{* *} p<0.01,{ }^{* * *} p<0.001$.

Additional file 4: Figure S4. Circ-GALNT16 could specifically interact with hnRNPK in the nucleus and suppress the proliferation and metastasis through binding to hnRNPK. a. MS analysis results of hnRNPK. b. The knockdown efficiency of hnRNPK. c. Pulldown and silver staining performed in nucleoprotein of DLD-1 cells. d, e. CCK8 and transwell showed that circ-GALNT16 suppressed the proliferation and metastasis of CRC by interacting with hnRNPK. $\mathrm{f}$. The mRNA expression level of hnRNPK in circGALNT16 knockdown and overexpression cells. All data are presented as the means $\pm \mathrm{SD}$ of three independent experiments. ${ }^{\mathrm{ns}} p>0.05,{ }^{*} p<0.05$, ${ }^{* *} p<0.01$

Additional file 5: Figure S5. Circ-GALNT16 promotes the interaction between hnRNPK and p53 via inhibiting SENP2-mediated deSUMOylation. a. SUMOylation modification analysis was performed to identify the levels of hnRNPK SUMOylation in circ-GALNT16 knockdown and overexpression cells. b. The co-IP assay showed SENP2 could interact with hnRNPK in CRC cells. c. The co-IP assay was performed between SENP2 and hnRNPK in circ-GALNT16 silencing and overexpression cells at $6 \mathrm{~h}$ after UV stimulation. $d$. The knockdown efficiency of SENP2. e. SUMOylation modification analysis to explore the levels of hnRNPK SUMOylation in circ-GALN T16 knockdown and SENP2 knockdown cells. f. Pulldown assay showed p53 did not physically interact with circ-GALNT16. g. CCK8 indicated that circ-GALNT16 suppressed the proliferation of CRC by attenuating the deSUMOylation of hnRNPK. All data are presented as the means \pm SD of three independent experiments. ${ }^{\text {ns }} p>0.05,{ }^{* *} p<0.01$.

Additional file 6: Figure S6. HnRNPK enhances the nuclear accumulation of circ-GALNT16. a. The mRNA expression level of GALNT16 in hnRNPK depletion cells. b-c. RNA FISH and subcellular fractionation assay again in hnRNPK-depletion cells. All data are shown as mean \pm SD of three independent experiments. ${ }^{\mathrm{ns}} p>0.05,{ }^{* *} p<0.01$.

Additional file 7: Figure S7. Circ-GALNT16 suppresses the proliferation ability of CRC cells through downregulating Serpine1. a. The knockdown 
and overexpression efficiency of Serpine1. b-g. CCK-8, colony formation, $\mathrm{EdU}$ assays were carried out in relatively treated cells targeted circ-GALN T16 and Serpine1. All data are shown as mean \pm SD of three independent experiments. ${ }^{*} p<0.05,{ }^{* *} p<0.01$.

Additional file 8: Figure S8. Circ-GALNT16 regulates cell cycle and apoptosis of CRC cells through downregulating Serpine1. a. Cell cycle assays were performed in circ-GALNT16 overexpression and Serpine1 overexpression groups. b. Cell apoptosis assays were performed in sh-circ-

GALNT16 and si-Serpine1 co-transfected cells. ${ }^{* *} p<0.01$

Additional file 9: Figure S9. Circ-GALNT16 suppresses the metastasis ability of CRC cells through Serpine1. a-d. Transwell and wound healing assays were performed in relatively treated cells targeted circ-GALNT16 and Serpine1. All data are shown as mean \pm SD of three independent experiments. ${ }^{*} p<0.05,{ }^{* *} p<0.01$

Additional file 10: Figure S10. Circ-GALNT16 promotes p21 mRNA expression level through the SUMOylation of hnRNPK and p53. a, b. The mRNA level of p21 in circ-GALNT16 knockdown and overexpression cells while hnRNPK was silenced. c. The expression of p21 in circ-GALNT16 knockdown cells while SENP2 was silenced. $d$. The expression of p21 in

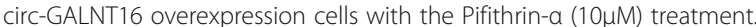
e, f. HnRNPK and p53 chromatin immunoprecipitation were performed to measure the hnRNPK and p53 enrichment at the promoter region(s) of p21 in DLD-1 and RKO. All data are shown as mean \pm SD of three independent experiments. ${ }^{\text {ns }} p>0.05,{ }^{* *} p<0.01$.

Additional file 11: Table S1. The differential expression circRNAs in CRC tissues according to the circRNA microarray.

Additional file 12: Table S2. The expression of circ-GALNT16 in 100 pairs of CRC tissues and relative expression in different pathological groups.

Additional file 13: Table S3. Primers, shRNAs, siRNAs, and probes used in this study.

Additional file 14: Table S4. Primary antibodies information used in this study.

Additional file 15: Table S5. Differential proteins identified by mass spectrometry in DLD-1 and LoVo and the list of RNA-binding proteins.

Additional file 16: Table S6. The mRNA sequencing results constructed in circ-GALNT16 overexpression and control RKO cells.

\section{Acknowledgements}

The authors thank Andy Zhao for her kindly supports during this study. We would like to thank the Core Facility of Jiangsu Provincial People's Hospital for its help in the detection of experimental samples.

\section{Authors' contributions}

Chaofan Peng, Wen Peng, Junwei Tang, and Yueming Sun generated the hypothesis and designed the study. Chaofan Peng, Yuqian Tan, Peng Yang performed experiments. Kangpeng Jin and Chuan Zhang performed animal experiments. Chaofan Peng, Yifei Feng, Lu Wang, Jiahui Zhou, Ranran Chen, Jiangzhou Ji, Tuo Wang, Chi Jin interpreted the data. Chaofan Peng wrote the manuscript. Yueming Sun and Junwei Tang supervised the overall research, secured funding, and interpreted results. The author(s) read and approved the final manuscript

\section{Funding}

This study was supported by Jiangsu Provincial Natural Science Foundation for Basic Research, China (Grant No. BK20201491), the National Key R\&D Program of China (2017YFC0908200), and Jiangsu Key Medical Discipline (General Surgery; Grant No. ZDxKA2016005).

\section{Availability of data and materials}

The data supporting the findings of this article is included within this article and its additional files.

\section{Declarations}

Ethics approval and consent to participate

This study was approved by the Ethics Committee of the First Affiliated Hospital with Nanjing Medical University.

\section{Consent for publication}

Not applicable.

\section{Competing interests}

The authors declare that they have no competing interests.

\section{Author details}

Department of General Surgery, The First Affiliated Hospital of Nanjing Medical University, Jiangsu 210029 Nanjing, People's Republic of China. ${ }^{2}$ The First School of Clinical Medicine, Nanjing Medical University, Nanjing, China. ${ }^{3}$ Nanjing Medical University, Nanjing, China.

Received: 7 May 2021 Accepted: 14 August 2021

Published online: 27 August 2021

\section{References}

1. Siegel RL, Miller KD, Goding Sauer A, Fedewa SA, Butterly LF, Anderson JC, et al. Colorectal cancer statistics, 2020. CA Cancer J Clin. 2020;70(3):145-64

2. Siegel RL, Miller KD, Jemal A. Cancer statistics, 2020. CA Cancer J Clin. 2020; 70(1):7-30.

3. Chen LL. The expanding regulatory mechanisms and cellular functions of circular RNAs. Nat Rev Mol Cell Biol. 2020;21(8):475-90.

4. Kristensen LS, Andersen MS, Stagsted LW, Ebbesen KK, Hansen TB, Kjems J. The biogenesis, biology and characterization of circular RNAs. Nat Rev Genet. 2019;20(11):675-91.

5. Yang H, Li X, Meng Q, Sun H, Wu S, Hu W, et al. CircPTK2 (hsa_circ_ 0005273) as a novel therapeutic target for metastatic colorectal cancer. Mol Cancer. 2020;19(1):13.

6. Su M, Xiao Y, Ma J, Tang Y, Tian B, Zhang Y, et al. Circular RNAs in Cancer: emerging functions in hallmarks, stemness, resistance and roles as potential biomarkers. Mol Cancer. 2019;18(1):90.

7. Jian X, He H, Zhu J, Zhang Q, Zheng Z, Liang X, et al. Hsa_circ_001680 affects the proliferation and migration of CRC and mediates its chemoresistance by regulating BMl1 through miR-340. Mol Cancer. 2020; 19(1):20.

8. Du WW, Yang W, Li X, Awan FM, Yang Z, Fang L, et al. A circular RNA circDNMT1 enhances breast cancer progression by activating autophagy. Oncogene. 2018:37(44):5829-42.

9. Liu Y, Ma C, Qin X, Yu H, Shen L, Jin H. Circular RNA circ_001350 regulates glioma cell proliferation, apoptosis, and metastatic properties by acting as a miRNA sponge. J Cell Biochem. 2019;120(9):15280-7.

10. Chen Z, Ren R, Wan D, Wang Y, Xue X, Jiang M, et al. Hsa_circ 101555 functions as a competing endogenous RNA of miR-597-5p to promote colorectal cancer progression. Oncogene. 2019;38(32):6017-34.

11. Zhao H, Chen S, Fu Q. Exosomes from CD133(+) cells carrying circ-ABCC1 mediate cell stemness and metastasis in colorectal cancer. J Cell Biochem. 2020:121(5-6):3286-97.

12. Zheng X, Chen L, Zhou Y, Wang Q, Zheng Z, Xu B, et al. A novel protein encoded by a circular RNA circPPP1R12A promotes tumor pathogenesis and metastasis of colon cancer via Hippo-YAP signaling. Mol Cancer. 2019; 18(1):47.

13. Wang Z, Qiu H, He J, Liu L, Xue W, Fox A, et al. The emerging roles of hnRNPK. J Cell Physiol. 2020;235(3):1995-2008.

14. Xu Y, Wu W, Han Q, Wang Y, Li C, Zhang P, et al. Post-translational modification control of RNA-binding protein hnRNPK function. Open Biol. 2019;9(3):180239.

15. Ritchie SA, Pasha MK, Batten DJ, Sharma RK, Olson DJ, Ross AR, et al. Identification of the SRC pyrimidine-binding protein (SPy) as hnRNP K: implications in the regulation of SRC1A transcription. Nucleic Acids Res. 2003:31(5):1502-13.

16. Lee SW, Lee MH, Park JH, Kang SH, Yoo HM, Ka SH, et al. SUMOylation of hnRNP-K is required for p53-mediated cell-cycle arrest in response to DNA damage. EMBO J. 2012;31(23):4441-52. 
17. Pelisch F, Pozzi B, Risso G, Munoz MJ, Srebrow A. DNA damage-induced heterogeneous nuclear ribonucleoprotein K sumoylation regulates p53 transcriptional activation. J Biol Chem. 2012;287(36):30789-99.

18. Moumen A, Masterson P, O'Connor MJ, Jackson SP. hnRNP K: an HDM2 target and transcriptional coactivator of p53 in response to DNA damage. Cell. 2005;123(6):1065-78.

19. Li J, Peng W, Yang P, Chen R, Gu Q, Qian W, et al. MicroRNA-1224-5p inhibits metastasis and epithelial-mesenchymal transition in colorectal cancer by targeting SP1-mediated NF-kappaB signaling pathways. Front Oncol. 2020;10:294.

20. Ding L, Zhao Y, Dang S, Wang Y, Li X, Yu X, et al. Circular RNA circ-DONSON facilitates gastric cancer growth and invasion via NURF complex dependent activation of transcription factor SOX4. Mol Cancer. 2019;18(1):45.

21. Zhang Z, Li J, Huang Y, Peng W, Qian W, Gu J, et al. Upregulated miR-1258 regulates cell cycle and inhibits cell proliferation by directly targeting E2F8 in CRC. Cell Prolif. 2018;51(6):e12505.

22. Yang P, Li J, Peng C, Tan Y, Chen R, Peng W, et al. TCONS_00012883 promotes proliferation and metastasis via DDX3/YY1/MMP1/PI3K-AKT axis in colorectal cancer. Clin Transl Med. 2020;10(6):e211.

23. Ji L, Li X, Zhou Z, Zheng Z, Jin L, Jiang F. LINC01413/hnRNP-K/ZEB1 Axis Accelerates Cell Proliferation and EMT in Colorectal Cancer via Inducing YAP1/TAZ1 Translocation. Mol Ther Nucleic Acids. 2020;19:546-61.

24. Peng W, Zhang C, Peng J, Huang Y, Peng C, Tan Y, et al. Lnc-FAM84B-4 acts as an oncogenic IncRNA by interacting with protein hnRNPK to restrain MAPK phosphatases-DUSP1 expression. Cancer Lett. 2020;494:94-106.

25. Qin G, Tu X, Li H, Cao P, Chen X, Song J, et al. Long noncoding RNA p53stabilizing and activating RNA promotes p53 signaling by inhibiting heterogeneous nuclear ribonucleoprotein K deSUMOylation and suppresses hepatocellular carcinoma. Hepatology. 2020;71(1):112-29.

26. Barysch SV, Dittner C, Flotho A, Becker J, Melchior F. Identification and analysis of endogenous SUMO1 and SUMO2/3 targets in mammalian cells and tissues using monoclonal antibodies. Nat Protoc. 2014;9(4):896-909.

27. Zhou WY, Cai ZR, Liu J, Wang DS, Ju HQ, Xu RH. Circular RNA: metabolism, functions and interactions with proteins. Mol Cancer. 2020;19(1):172.

28. Bomsztyk K, Denisenko O, Ostrowski J. hnRNP K: one protein multiple processes. Bioessays. 2004;26(6):629-38.

29. Ostrowski J. Transient recruitment of the hnRNP K protein to inducibly transcribed gene loci. Nucleic Acids Res. 2003;31(14):3954-62.

30. Expert-Bezancon A, Le Caer JP, Marie J. Heterogeneous nuclear ribonucleoprotein ( $h n R N P$ ) $\mathrm{K}$ is a component of an intronic splicing enhancer complex that activates the splicing of the alternative exon $6 \mathrm{~A}$ from chicken beta-tropomyosin pre-mRNA. J Biol Chem. 2002;277(19): 16614-23.

31. Lubelsky Y, Ulitsky I. Sequences enriched in Alu repeats drive nuclear localization of long RNAs in human cells. Nature. 2018;555(7694):107-11.

32. Xu Y, Chen W, Liang J, Zeng X, Ji K, Zhou J, et al. The miR-1185-2-3pGOLPH3L pathway promotes glucose metabolism in breast cancer by stabilizing p53-induced SERPINE1. J Exp Clin Cancer Res. 2021;40(1):47.

33. Bertoli C, Skotheim JM, de Bruin RA. Control of cell cycle transcription during G1 and S phases. Nat Rev Mol Cell Biol. 2013;14(8):518-28.

34. Adams JM, Cory S. The $\mathrm{BCl}-2$ apoptotic switch in cancer development and therapy. Oncogene. 2007;26(9):1324-37.

35. Bi J, Liu H, Dong W, Xie W, He Q, Cai Z, et al. Circular RNA circ-ZKSCAN1 inhibits bladder cancer progression through miR-1178-3p/p21 axis and acts as a prognostic factor of recurrence. Mol Cancer. 2019;18(1):133.

36. Zhang C, Zhang C, Lin J, Wang H. Circular RNA Hsa_Circ_0091579 serves as a diagnostic and prognostic marker for hepatocellular carcinoma. Cell Physiol Biochem. 2018;51(1):290-300.

37. Guo G, Wang H, Ye L, Shi X, Yan K, Lin K, et al. Hsa_circ_0000479 as a novel diagnostic biomarker of systemic lupus erythematosus. Front Immunol. 2019;10:2281.

38. Yang F, Fang E, Mei H, Chen Y, Li H, Li D, et al. Cis-Acting circ-CTNNB1 Promotes beta-Catenin Signaling and Cancer Progression via DDX3Mediated Transactivation of YY1. Cancer Res. 2019:79(3):557-71.

39. Chen $L Y$, Wang $L$, Ren $Y X$, Pang Z, Liu $Y$, Sun $X D$, et al. The circular RNA circERBIN promotes growth and metastasis of colorectal cancer by miR-125a-5p and miR-138-5p/4EBP-1 mediated cap-independent HIF-1alpha translation. Mol Cancer. 2020;19(1):164

40. Xue D, Wang H, Chen Y, Shen D, Lu J, Wang M, et al. Circ-AKT3 inhibits clear cell renal cell carcinoma metastasis via altering miR-296-3p/E-cadherin signals. Mol Cancer. 2019;18(1):151.
41. Wong CH, Lou UK, Li Y, Chan SL, Tong JH, To KF, et al. CircFOXK2 Promotes Growth and Metastasis of Pancreatic Ductal Adenocarcinoma by Complexing with RNA-Binding Proteins and Sponging MiR-942. Cancer Res. 2020;80(11):2138-49.

42. Zeng C, Chen Y. HTR1D, TIMP1, SERPINE1, MMP3 and CNR2 affect the survival of patients with colon adenocarcinoma. Oncol Lett. 2019;18(3): 2448-54.

43. Li S, Wei X, He J, Tian X, Yuan S, Sun L. Plasminogen activator inhibitor-1 in cancer research. Biomed Pharmacother. 2018;105:83-94.

44. Szoltysek K, Janus P, Zajac G, Stokowy T, Walaszczyk A, Widlak W, et al. RRAD, IL4I1, CDKN1A, and SERPINE1 genes are potentially co-regulated by NF-kappaB and p53 transcription factors in cells exposed to high doses of ionizing radiation. BMC Genom. 2018;19(1):813.

\section{Publisher's Note}

Springer Nature remains neutral with regard to jurisdictional claims in published maps and institutional affiliations.

\section{Ready to submit your research? Choose BMC and benefit from:}

- fast, convenient online submission

- thorough peer review by experienced researchers in your field

- rapid publication on acceptance

- support for research data, including large and complex data types

- gold Open Access which fosters wider collaboration and increased citations

- maximum visibility for your research: over $100 \mathrm{M}$ website views per year

At $\mathrm{BMC}$, research is always in progress.

Learn more biomedcentral.com/submissions 